\title{
La mise en eau du barrage hydroélectrique de Petit-Saut (Guyane): ses conséquences sur les peuplements d'invertébrés aquatiques et sur la nourriture des poissons
}

\author{
Filling of Petit-Saut hydroelectric reservoir \\ (French Guiana): impact on aquatic invertebrates \\ and fish nutriment
}

\author{
V. Horeau (1), $\mathrm{Ph}$. Cerdan ${ }^{(1)}$ et A. Champeau(2) \\ (1) Laboratoire Environnement de Petit-Saut, HYDRECO, 97388 Kourou cedex, Guyane fran- \\ çaise. \\ (2) Laboratoire d'hydrobiologie, Université de Provence, 13331 Marseille cedex.
}

Résumé. - La construction du barrage hydroélectrique de Petit-Saut sur le fleuve Sinnamary entraîne des modifications brutales. L'écosystème forestier et fluviatil devient un écosystème lacustre. Dès la mise en eau de la retenue (janvier 1994), une faune lacustre dense et diversifiée se développe dans l'épilimnion oxygéné. Par rapport aux invertébrés de la rivière, la richesse spécifique chute mais reste néanmoins élevée. La colonisation s'effectue par une succession d'espèces de petites tailles, détritivores, (cladocères Bosminidae et cyclopoïdes) puis d'espèces de plus grandes tailles, phytoplanctonophages (cladocères Daphniidae et calanoïdes), ensuite apparaissent les carnassiers (Chaoboridae) et enfin des ostracodes qui dominent largement la faune lacustre à la fin du remplissage (septembre 1995). Des macroinvertébrés benthiques (odonates, trichoptères,...) se développent sur les troncs et les branches immergés. Les poissons consomment cette nouvelle faune lacustre ainsi que les invertébrés terrestres ennoyés.

A l'aval du barrage, la faune d'invertébrés aquatiques du fleuve s'est modifiée. Dans la masse d'eau, elle est composée du plancton de la retenue entraîné par les eaux restituées. Dans les fonds sableux, les invertébrés aquatiques profitent de l'enrichissement en matière organique apportée par les eaux du lac.

Mots-clés. - Invertébrés aquatiques, fleuve Sinnamary, Petit-Saut, barrage hydroélectrique, mise en eau. 


\begin{abstract}
Construction of Petit-Saut hydroelectric dam on the Sinnamary river generated major modifications. The forest and fluvial ecosystem became a lacustrine ecosystem. Following the filling of the reservoir (January 1994), a dense and diversified lacustrine fauna developed in the oxygenated epilimnion. In comparison with invertebrates in the river, species diversity dropped but nonetheless remained high. Colonization was first by smaller species and detritivores (cladocera Bosminidae and Cyclopidae), next by larger phytoplankton feeders, (cladocera Daphniidae and Calanidae), later carnivores (Chaoboridae) and finally by ostracoda, which dominated at the end of the filling stage (September 1995). Benthic macroinvertebrates (dragonflies, stoneflies, etc.) developed on the immersed trunks and boughs. Fish feed on this new lacustrian fauna and land invertebrates trapped by inflow.

Downstream, the aquatic invertebrate fauna of the river has changed and is composed of plankton entrained from the reservoir. On the sandy bottom, benthic fauna benefit from the enrichment brought by lacustrine waters.
\end{abstract}

Key Words. - Aquatic invertebrates, Sinnamary river, Petit Saut, hydroelectric dam, filling phase.

\section{INTRODUCTION}

La faune aquatique notamment celle des invertébrés est fortement modifiée par la construction des barrages hydroélectriques. Dans les régions tempérées, de nombreuses études ont été réalisées sur les impacts subis par les peuplements d'invertébrés aquatiques lors d'aménagements effectués sur des cours d'eau (Grégoire, 1982; Bournaud et al., 1987; Décamps et al., 1987; Ward \& Stanford, 1987; Cereghino \& Lavaraller, 1996; Dumont et al., 1996, Parasiewicz et al., 1996). En Amazonie de nombreux fleuves ont été aménagés. Malheureusement, les données hydrobiologiques concernent soit l'état initial du site soit son évolution après la perturbation. De plus, ces résultats sont souvent inaccessibles faute d'avoir été publiés dans des revues scientifiques. Néanmoins des études sur Brokopondo au Surinam (Heide, 1982) et sur quelques lacs brésiliens comme Balbina (Falótico, 1993) nous permettent de mieux appréhender le devenir des peuplements d'invertébrés aquatiques dans la retenue de Petit-Saut.

Le barrage est construit sur le fleuve Sinnamary dont le bassin versant est de $5927 \mathrm{~km}^{2}$ et le débit moyen de $260 \mathrm{~m}^{3} \cdot \mathrm{s}^{-1}$. De 1991 à 1993, nous avons effectué un point zéro sur la faune d'invertébrés aquatiques dans le fleuve et ses rapides (localement appelés sauts), dans ses affluents (criques), dans des zones de forêt inondable ainsi que dans des marécages (pripris) du réseau hydrographique. Tous ces biotopes ont été prospectés en amont, en aval et dans la future retenue, en utilisant plusieurs méthodes de prélèvements en fonction des stations pour avoir une bonne représentation des différents groupes (Horeau, 1996). Malgré les problèmes taxonomiques rencontrés, la richesse spécifique est élevée chez les quelques groupes qui ont fait l'objet d'une 
étude systématique plus poussée comme les cladocères, les éphéméroptères ou les odonates (Horeau \& Richard, 1992). Contrairement aux fleuves des régions tempérées composés de trois zones successives. (crénon, rhytron et potamon), le fleuve Sinnamary se présente comme une succession de sauts ou rhytron (photographie A) et de zones plus calmes ou potamon appelées "entre-sauts". Les sauts présentent des substrats rocheux majoritairement colonisés par des larves d'insectes alors que les zones de potamon à substrat sableux sont colonisées par des crustacés psammophiles. Les deux communautés, très différentes, se succèdent de la source jusqu'à l'estuaire sans que chacune d'elles n'évolue sur le profil en long. Les peuplements d'invertébrés sont également différents dans les zones marécageuses annexes inondées en périodes pluvieuses dans lesquelles les microcrustacés sont mieux représentés. Dans les affluents, les invertébrés aquatiques varient en fonction du courant et des substrats.

Dans les différents biotopes, les communautés d'invertébrés aquatiques varient en fonction des saisons. Leur densité dépend principalement des variations de débits. Généralement, on observe les plus fortes densités en saison sèche et les plus faibles en saison des pluies, après dilution (excepté pour certains organismes comme les cladocères détritivores qui sont favorisés par la charge plus élevée en matières en suspensions (M.E.S.) pendant la période de hautes eaux). Cette réparti- tion est affectée dans plusieurs stations du Courcibo (principal affluent du fleuve Sinnamary, photographie B) par les activités d'orpaillage qui engendrent d'énormes quantités de M.E.S. rendant la vie impossible pour la majorité des espèces.

Enfin, avec les nombreux éléments provenant de la forêt (feuilles, fleurs, graines et invertébrés terrestres), les invertébrés aquatiques contribuent à l'alimentation de certains poissons (Horeau, 1996; Horeau et al., 1996a). Si dans les criques, où le couvert forestier est proche de $100 \%$, les poissons consomment essentiellement des invertébrés terrestres, leur nourriture est principalement constituée d'invertébrés aquatiques dans les sauts.

La retenue de Petit-Saut (photographies $C$ et D) dont la profondeur maximale est de 35 mètres et la profondeur moyenne de 10-11 mètres recouvre $310 \mathrm{~km}^{2}$ de forêt primaire. Depuis le début de la mise en eau, nous continuons le suivi des invertébrés aquatiques pour évaluer la qualité biologique des eaux de la retenue et de son tronçon aval. Nous pourrons ainsi apporter des éléments de réponse aux nombreuses questions qui se posent dans ce nouvel écosystème;

- quels sont les invertébrés aquatiques qui se développent dans le lac?

- leur développement est-il soumis à des paramètres abiotiques comme la qualité physico-chimique des eaux ou les variations saisonnières et biotiques comme le dévelop- 

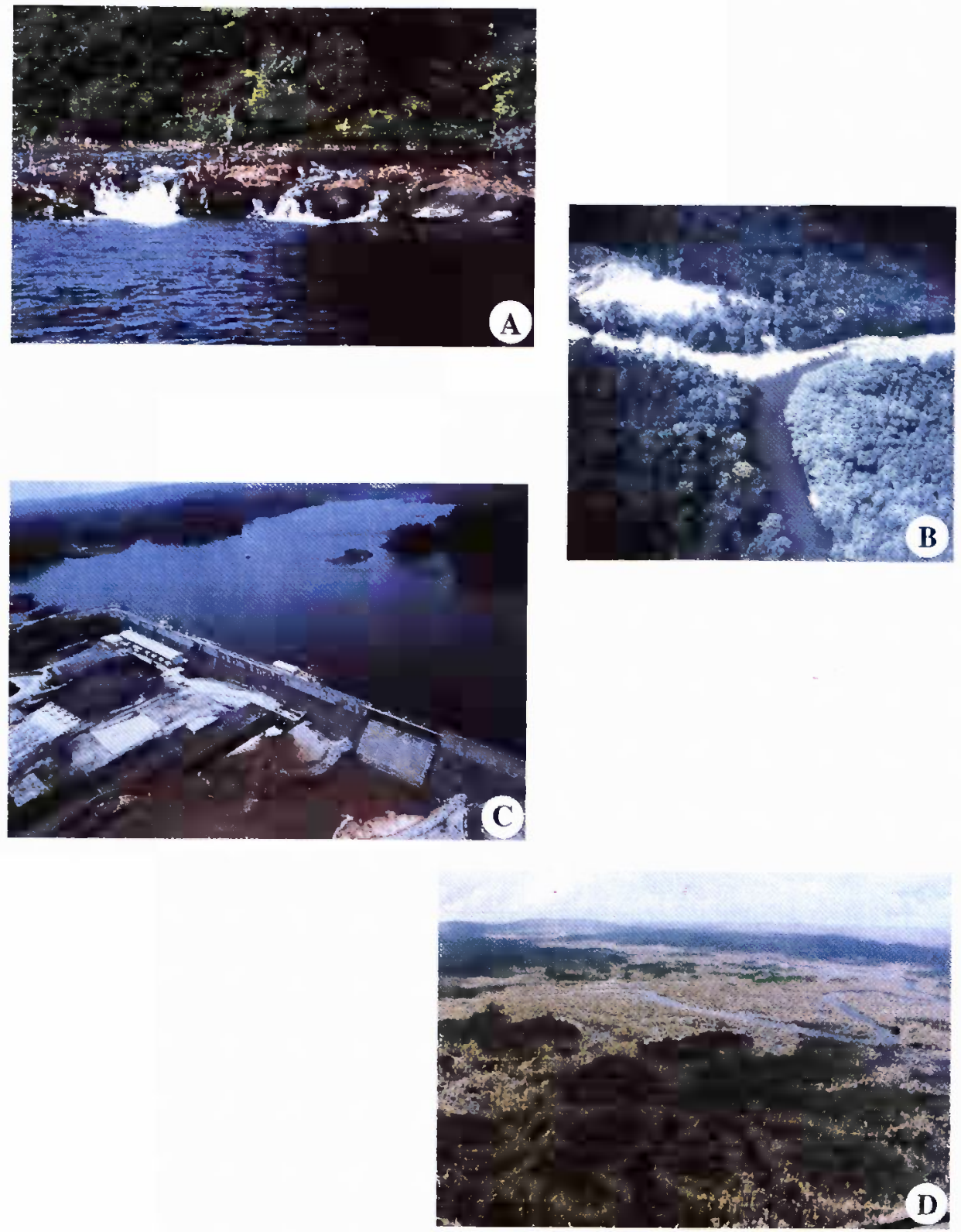

Planche photographique. - Quelques vues du fleuve Sinnamary et de la retenue de Petit-Saut. (A) : Un saut du fleuve Sinnamary avec de nombreux substrats rocheux colonisés par des podostémacées (forte pente).

(B): Deux Branches; confluence du Courcibo (eaux chargées en matières en suspention, conséquences de l'activité aurifère) et du Sinnamary.

(C) : Vue aérienne de l'ouvrage.

(D) : Vue aérienne du plan d'eau à proximité de l'ouvrage avec la zone axiale (ancien lit du fleuve), la forêt inondée caractérisée par les arbres morts et les nombreuses iles non atteintes par les eaux. Photographic plates. - Some views of Sinnamary River and Petit-Saut dam.

(A) : A Sinnamary River's fall with substrate rocks colonised by podostemacea (big incline).

(B) : Deux Branches; confluence of Courcibo River (water with many suspended matters, result of gold mining activities) and Sinnamary River.

(C): Aerial view of the dam.

(D) : Aerial view of the reservoir near the dam :

- the axial zone (old bed of the river);

- the flooded forest caracterised by dead trees;

- islands no reached by the inflow. 
pement du phytoplancton, la présence de prédateurs,...?

- les poissons du lac vont-ils utiliser cette nouvelle ressource alimentaire?

- à l'aval, les invertébrés aquatiques en place se maintiennent-ils ou sont-ils remplacés?

- la faune à l'aval est-elle influencée par les eaux du lac et par la gestion du barrage?

\section{MATÉRIEL ET MÉTHODES}

Dans la retenue, depuis la mise en eau, la faune pélagique est collectée à l'aide de filets (diamètre $=30 \mathrm{~cm}$, hauteur $=70 \mathrm{~cm}$ et vide de maille $=$ $50 \mu \mathrm{m}$ et $100 \mu \mathrm{m})$. Les densités sont obtenues par comptage d'échantillons effectués sur des colonnes d'eau. Les résultats sont exprimés en nombre d'individus par unité de surface (ind. $/ \mathrm{m}^{2}$ ). La faune benthique est collectée à l'aide de substrats constitués de feuilles et de blocs granitiques (d'environ $20 \mathrm{~cm}$ de long, $10 \mathrm{~cm}$ de large et $10 \mathrm{~cm}$ de hauteur). Ces prélèvements lacustres sont réalisés, en zone axiale ou déboisée et en zone boisée, aux sites de Petit-Saut, Roche Génipa et Crique Bonne Nouvelle (carte $\mathrm{N}^{\circ} 1$ ).

Carte 1. - Localisation des stations d'échantillonnage en amont, dans le réservoir et en aval du barrage de Petit-Saut. (1-11: cf tableau IV) Map 1. - Location of sampling stations upstream, in Petit-Saut dam reservoir and downstream. (1-11: cf. table IV)

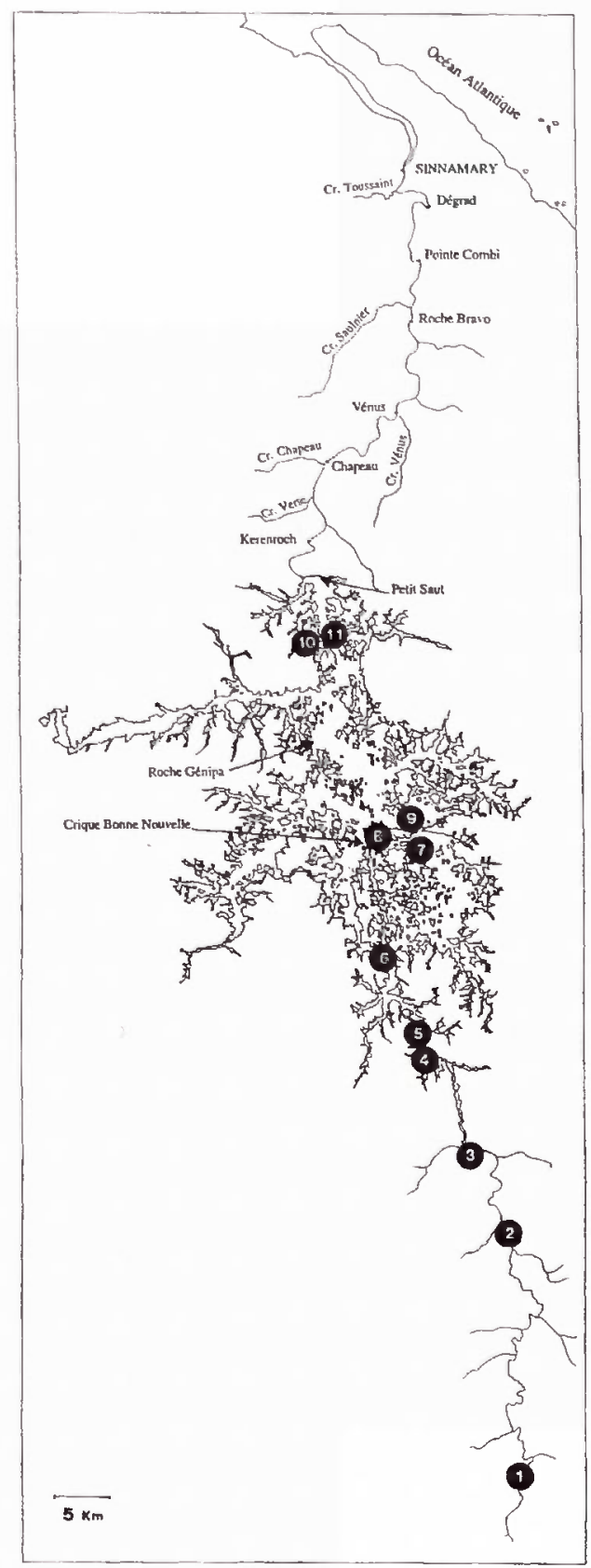


Lors de missions communes avec les ichtyologues du centre O.R.S.T.O.M. de Cayenne, des poissons sont collectés avec une batterie de 10 filets maillants (longueur: $25 \mathrm{~m}$, hauteur : $2,5 \mathrm{~m}$ ) de mailles croissantes entre 10 et 60 $\mathrm{mm}$ de vide de maille, placés dans le lac, au niveau du front (zone entre le lac et la rivière) ainsi qu'en amont du lac. Les sites de prélèvement situés en amont de l'ouvrage $\left(n^{\circ} 1\right.$ à 11$)$ sont reportés sur la carte $\mathrm{N}^{0} 1$. Chaque poisson est identifié, mesuré et pesé. Leur estomac est prélevé et conservé dans de l'alcool à 70\%. Au laboratoire, l'observation des estomacs s'effectue sous loupe binoculaire. II s'agit de reconnaître le matériel ingéré et de le quantifier. La quantification s'effectue par volumétrie, à l'aide de cuves de Dollfus. Ces cuves sont constituées de 200 cases identiques $(0,5 \mathrm{~cm}$ de côté). Le volume de chaque aliment est estimé par rapport au nombre de cases qu'il occupe dans cette cuve. Les résultats sont donc exprimés en volume ou en pourcentage volumétrique.

A l'aval (les stations d'études sont reportées sur la carte $\mathrm{N}^{\circ} 1$ ), les invertébrés de la masse d'eau sont collectés avec les mêmes filets à plancton déjà utilisés dans le lac, mais munis de flowmeter pour évaluer le volume d'eau échantillonné. Les résultats sont exprimés en nombre d'individus par unité de volume (ind./I). La faune benthique est collectée avec une drague. Au laboratoire, le comptage s'effectue sur un litre de sable à partir duquel on extrait la faune par trois rinçages successifs. Les résultats sont alors exprimés en nombre d'individus par unité d'échantillonnage.

\section{RÉSULTATS ET DISCUSSION}

\subsection{La retenue}

Les différents biotopes qui constituent le réseau hydrographique (sauts, entre-sauts, criques,...) disparaissent pour ne laisser place qu'à un biotope d'eau plus ou moins stagnante sur l'ensemble de la retenue. Le suivi des invertébrés montre trois points.

- La biocénose d'eau courante disparaît dès le début de la mise en eau et la biomasse lacustre s'installe très rapidement, en quelques semaines, avec l'essor d'un zooplancton abondant, diversifié et essentiellement pélagique.

- Une faune benthique se développe également sur les nombreux supports de la forêt inondée.

- Les poissons de la retenue consomment ces nouvelles ressources.

\subsubsection{La faune pélagique}

La mise en eau de la retenue a diminué la richesse spécifique. Par exemple, le nombre d'espèces de cladocères qui est de 43 avant la mise en eau (Horeau, 1996), est divisé par quatre dans la retenue. De même, sur la retenue, nous n'observons plus les 76 espèces d'odonates dénombrées sur l'ensemble du bassin versant du fleuve Sinnamary, avant la mise en 
eau (Horeau et al., 1996b). Néanmoins, la richesse spécifique reste élevée. On dénombre à ce jour 87 espèces de rotifères (Pourriot, 1996) qui figurent dans le tableau I. Une espèce est nouvelle pour la science (Segers et Pourriot, 1997), d'autres présentent des originalités au niveau infra-spécifique (Pourriot et al., 1997). On distingue une dizaine d'espèces de cladocères et 7 espèces de copépodes dont une espèce de calanoïde du genre Notodiaptomus est une espèce nouvelle pour la science (tableau II).

\subsection{1.a Répartition verticale de la faune pélagique}

La stratification très rapide du $\mathrm{mi}$ lieu avec une thermocline et une oxy- cline peu profonde (Richard, 1996) implique une distribution de la faune aquatique essentiellement cantonnée dans l'épilimnion. Effectivement, à l'exception de quelques Chaoboridae, le zooplancton est exclu de l'hypolimnion anoxique (fig. 1). Ceci s'explique par des exigences physiologiques chez ces organismes qui "respirent» l'oxygène dissous dans l'eau. Les pics de densité sont observés à la limite entre l'épilimnion et l'hypolimnion. Ces deux masses d'eau sont séparées par une différence de densité occasionnée par la température des masses d'eau; les eaux de surface sont chaudes (entre $30^{\circ} \mathrm{C}$ en surface et $29,5^{\circ} \mathrm{C}$ à $-5 \mathrm{~m}$ ) alors que les eaux hypolimniques sont "froides» (entre
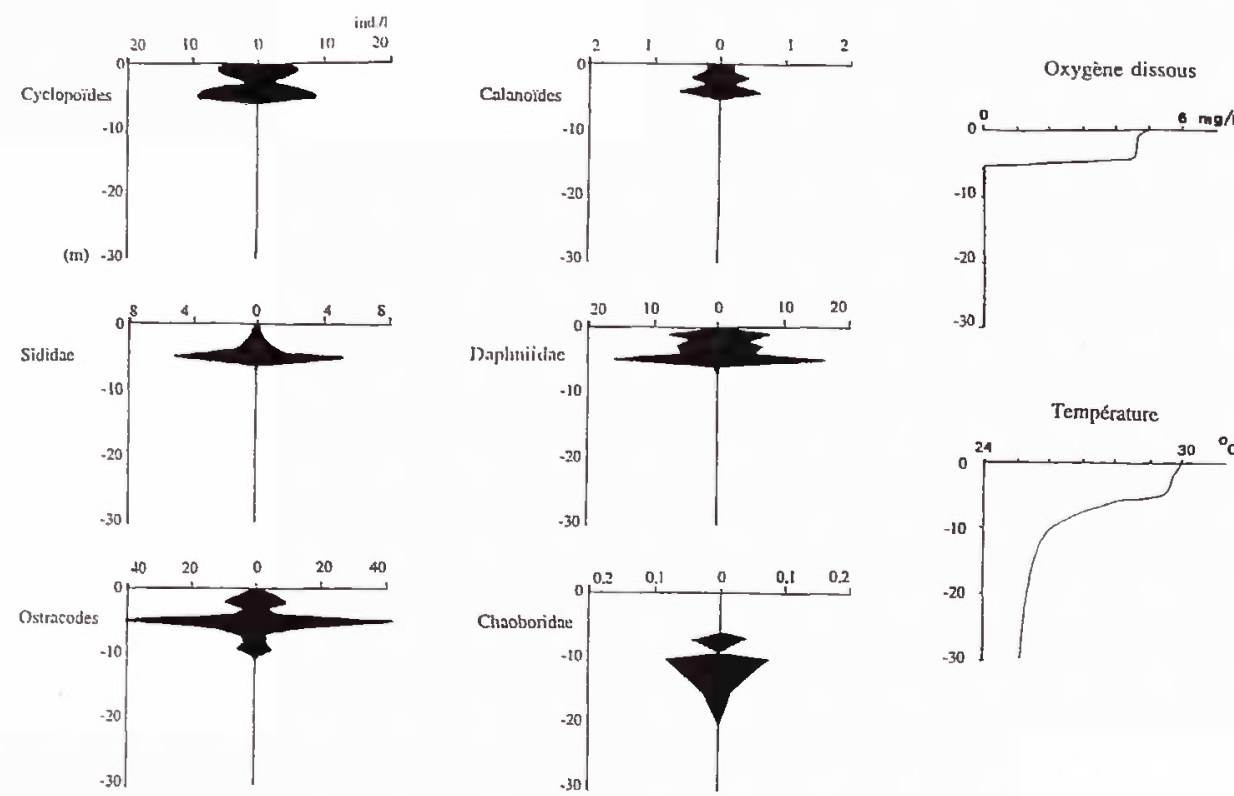

Fig. 1. - Répartition verticale des principaux éléments du zooplancton en relation avec l'oxygène dissous et la température de la masse d'eau, dans la station axiale de Roche Génipa (pK 23,6), le $05-X \mid 1-96$.

Fig. 1. - Vertical distribution of the zooplankton in relation with dissolved oxygen and temperature of water, in axial station of Roche Génipa ( $\mathrm{pK} 23,6$ ), on the 5-12-96. 
Tableau I. - Rotifères collectés dans la retenue de Petit Saut (Pourriot, 1996). Table I. - Rotifers collected in Petit Saut Dam (Pourriot, 1996).

\author{
Anuraeopsis fissa (Gosse) \\ Ascomorpha ecaudis (Perty) \\ Ascomorpha saltans Bartsch \\ Asplanchna intermedia \\ Brachionus patulus (Müller) \\ Brachionus quadridentatus Hermann \\ Cephalodella gibba (Ehrenberg) \\ Cephalodellasp. \\ Colurella colurus (Ehrenberg) \\ Colurella obtusa (Gosse) \\ Colurella uncinata (Müller) \\ Conochiloides coenobasis Skorikov \\ Conochilus cf. hippocrepis (Schrank) \\ Dicranophorus epicharis Harring \& Myers \\ Dicranophorus prionacis Harring \& Myers \\ Dipleuchlanis propatula (Gosse) \\ Epiphanes claumala Ehrbenberg \\ Euchlanis lyra Hudson \\ Euchlanis semicarinata Sergers \\ Filimia novaezealandiae Shiel \& Sanoamuang \\ Filinia saltator (Gosse) \\ Hexarthra longicomicula Tumer \\ Keratella lenzi (Hauer) \\ Lecane bulla (Gosse) \\ Lecane closterocerca (Schmarda) \\ Lecane copeis Harring \& Myers \\ Lecane comuta (Müller) \\ Lecane cunvicomis (Muray) \\ Lecane sp. nov. Segers \& Pourriot \\ Lecane eutarsa Harring \& Myers \\ Lecane flexilis (Gosse) \\ Lecane furcata (Murray) \\ Lecane haliclysta Harring \& Myers \\ Lecane hamata hamata (Stokes) \\ Lecane inopinata Harring \& Myers \\ Lecane lauterbomi Hauer \\ Lecane leontina (Tumer) \\ Lecane ludwigiif. lacinulata Hauer \\ Lecane ludwigii f. ohioensis (Herrick) \\ Lecane ludwigii f. typica (Eckstein)) \\ Lecane lunaris (Ehrbenberg) \\ Lecane monostyla (Daday) \\ Lecane obtusa (Murray)
}

Lecane quadridentata (Ehibenberg)

Lecane signifera (Jennings)

Lecane stichaea Harring

Lecane syngenes (Hauer)

Lepadella donneri Koste

Lepadella ovalis (Müller)

Lepadella patella (Müller)

Lepadella quinquecostata (Lucks)

Lepadella costatoides Segers

Lepadella rhomboides f. imbricata (Harring)

Monnomata cf. grandis Tessin

Mytilina acantophora Hauer

Mytili (Lucks)

Mytilina crassipes (Lucks)

Mytilina lobata sp. nov.

Mytilina macrocera (Jennings)

Mytilina michelangellii Reid \& Turner

Mytilina unguipes (Lucks)

Mytilina ventralis (Ehrbenberg)

Mytilina ventralis f. macracantha (Gosse)

Notommata aurita (Müller)

Notommata cerberus Gosse

Notommata copeus Ehrbenberg

Platyias leloupi (Gillard)

Platyias quadricomis (Ehrbenberg)

Platyias quadricornis $f$. brevispinus Daday

Ploesoma lenticulare Herrick

Polyarthra major Burckhardt

Polyarthra vulgaris Carlin

Resticula cf. melandocus (Gosse)

Rotaria rotatoria Pallas

Sinantherina sp.

Testudinella ohlei Koste

Testudinella incisa f. emerginula (Stenroos)

Testudinella mucronata (Gosse)

Testudinella patina (Hermann)

Testudinella tridentata amazonica Thomasson

Trichocerca bicristata (Gosse)

Trichocerca inemis (Linder)

Trichocerca pusilla (Jennings)

Trichocerca similis (Wierzejski)

Trichocerca tenuior (Gosse)

Trichocerca tigris (Müller)

Trichocerca tetractis (Ehrbenberg) 
Tableau II. - Invertébrés pélagiques (autres que les rotifères) collectés dans la retenue de Petit Saut. [ ]. - espèce dominante dans la famille correspondante.

Table II. - Pelagics invertebrate (other than rotifers) collected in Petit Saut dam. [ ]. dominante specie in the family.

\begin{tabular}{|c|c|c|c|}
\hline Classe & Famille & Espèce & Déterminateur \\
\hline \multirow[t]{5}{*}{ Copépodes } & Diaptomidae & $\begin{array}{l}\text { Notodiaptomus echinatus (Lowndes, 1934) } \\
\text { Notodiaptomus sp. nov. }\end{array}$ & $\begin{array}{c}\text { Defaye, } 1995 \\
"\end{array}$ \\
\hline & Cyclopidae & Metacyclops hartmanni Herst, 1960 & " \\
\hline & & Thermocyclops minurus (Lowndes, 1934) & " \\
\hline & & Mesocyclops meridianus (Kiefer, 1926) & $"$ \\
\hline & & Mesocyclops ellipticus Kiefer, 1936 & $"$ \\
\hline \multirow[t]{5}{*}{ Cladocères } & Bosminidae & [Bosminopsis deiteirsii] & \\
\hline & Daphniidac & [Ceriodaphnia comuta] & \\
\hline & Sididae & & \\
\hline & Macrothricidae & & \\
\hline & Chydoridae & & \\
\hline Ostracodes & Candonidae & Physocypria affinis Klie, 1933 & Martens, 1995 \\
\hline Diptères & Chaoboridae & Chaoborus sp & \\
\hline
\end{tabular}

$28,9^{\circ} \mathrm{C}$ à $-5,5 \mathrm{~m}$ et $25,1^{\circ} \mathrm{C}$ au fond). Les organismes constamment soumis à la sédimentation trouvent au niveau de la thermocline et de l'oxycline une barrière physique qui les aident à se maintenir en limite de la zone oxique. Contrairement à la répartition des autres microcrustacés, celle des ostracodes s'étale jusqu'à $-10 \mathrm{~m}$, avec des densités relativement élevées jusqu'à $-9 \mathrm{~m}$. Cette localisation plus profonde que celle des cladocères et des copépodes révèle leurs préférences benthiques.

\subsection{1.b Evolution saisonnière de la faune pélagique}

Comme pour la physico-chimie des eaux (Richard, 1997), durant la phase de remplissage, la station médiane
Roche Génipa, représente l'état de structuration le plus avancé et le plus stable du lac. Dans le temps, on constate une succession d'espèces de petites tailles, détritivores, stratège $\mathrm{R}$, avant le "bloom » phytoplanctonique, puis d'espèces de plus grandes tailles, stratège $\mathrm{K}$ (Horeau, 1996), après le développement du phytoplancton (Vaquer, 1997). Chez les cladocères (fig. 2), durant la phase de remplissage, on passe d'un peuplement essentiellement constitué de Bosminidae (détritivores) à un peuplement dominé par les Daphniidae (phytophages). Chez les copépodes (fig. 3), le schéma est similaire: on passe d'un peuplement essentiellement constitué de cyclopoïdes à un peuplement dominé par les calanoïdes phytoplanctonophages après 


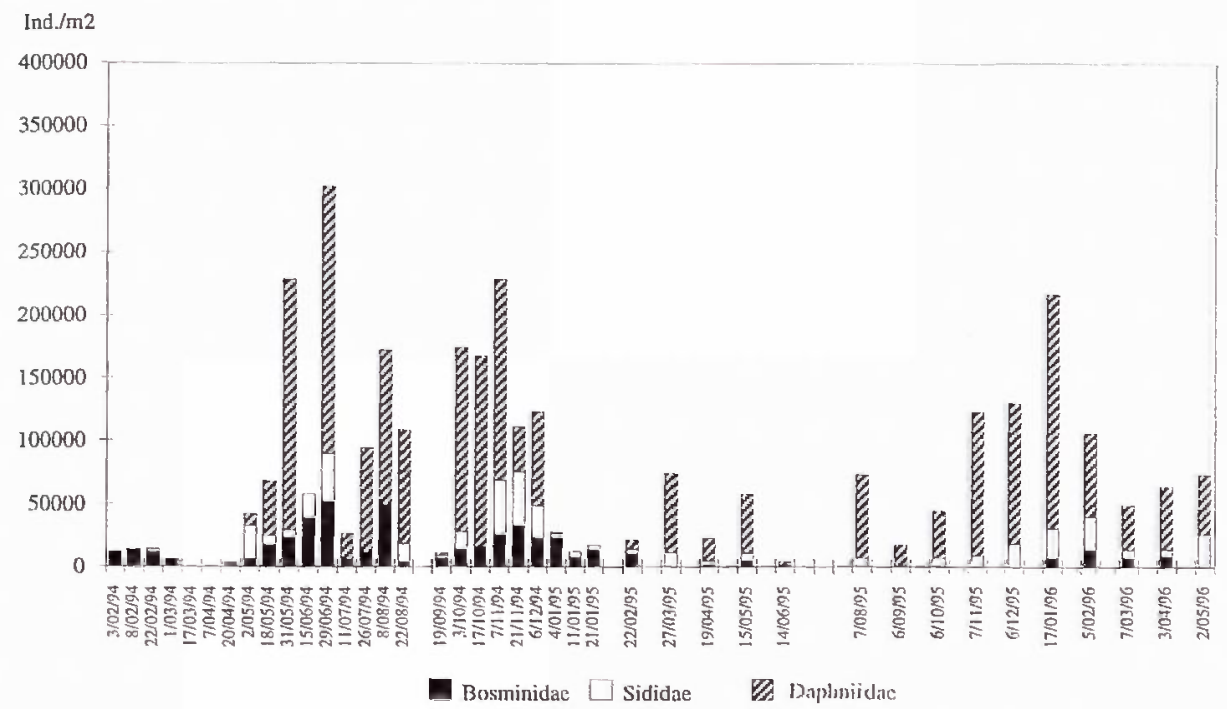

Fig. 2. - Evolution des cladocères depuis le début du remplissage (janvier 1994 - juin 1996), à Roche Génipa 1.

Fig. 2. - Roche Génipa 1 station. - development of cladocera from the beginning of the filling phase (January 1994 - June 1996).

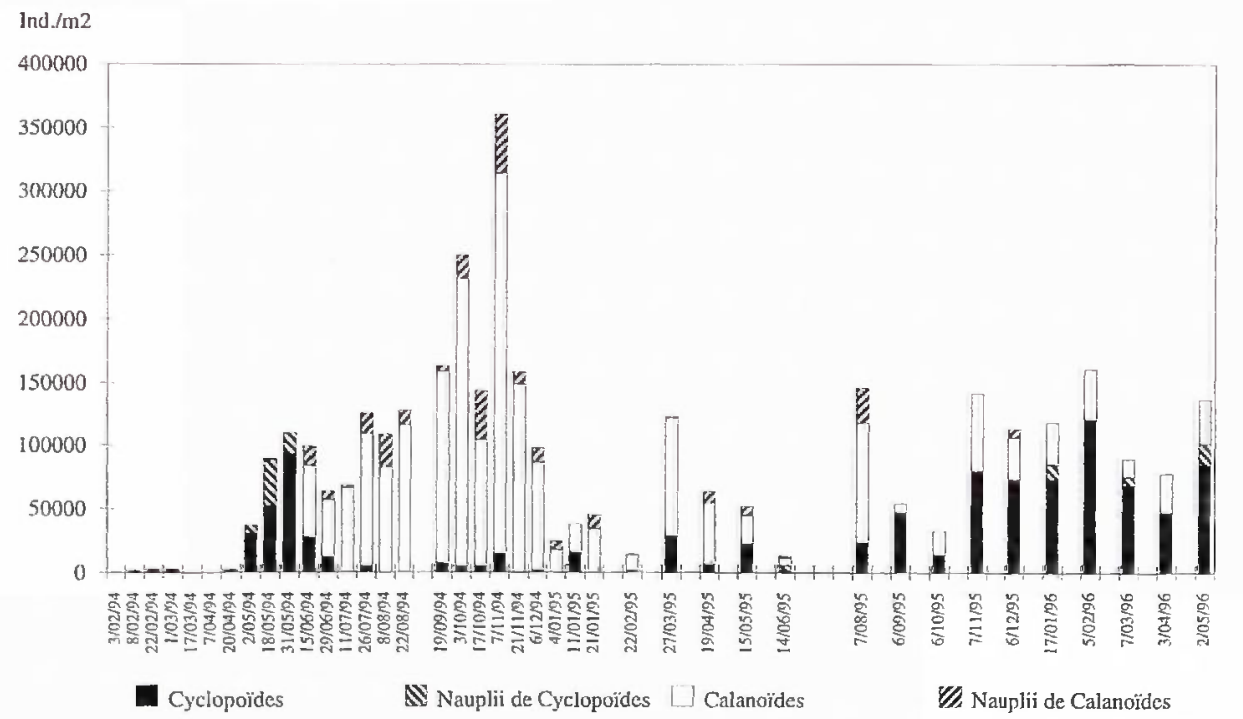

Fig. 3. - Evolution des copépodes depuis le début du remplissage (janvier 1994 - juin 1996), à Roche Génipa 1.

Fig. 3. - Roche Génipa 1 station. - development of copepoda from the beginning of the filling phase (January 1994 - June 1996). 
le développement du phytoplancton. A Roche Génipa, 6 mois après le début de la mise en eau, on observe une représentation dominante des calanoïdes, indicateurs du bon fonctionnement de l'épilimnion du lac.

En février 95, des ostracodes sont observés au site de Petit Saut. Habituellement, les ostracodes sont benthiques. Comme à Petit Saut l'hypolimnion est anoxique, leur développement pourrait donc sembler impossible. Mais, il s'agit de Physocypria affinis qui est une espèce semi-planctonique, munie de longs poils natatoires sur les antennes qui lui permettent de très bien nager. Depuis septembre 1995, cet ostracode domine le zooplancton des trois sites étudiés : Petit-Saut, Roche Génipa et Crique Bonne Nouvelle.

La maturité du système peut être gravement perturbée (fig. 4) lors d'épisodes très pluvieux comme à la fin de l'année 1994 ou au début de l'année 1996 (début de la saison des pluies) qui engendrent un mélange partiel de la masse d'eau avec baisse de l'oxygène dissous et remontée de débris organiques. Dans ces situations éphémères, le zooplancton est en parti éradiqué mais se reconstitue rapidement selon la même succession, plus ou moins tronquée, que lors

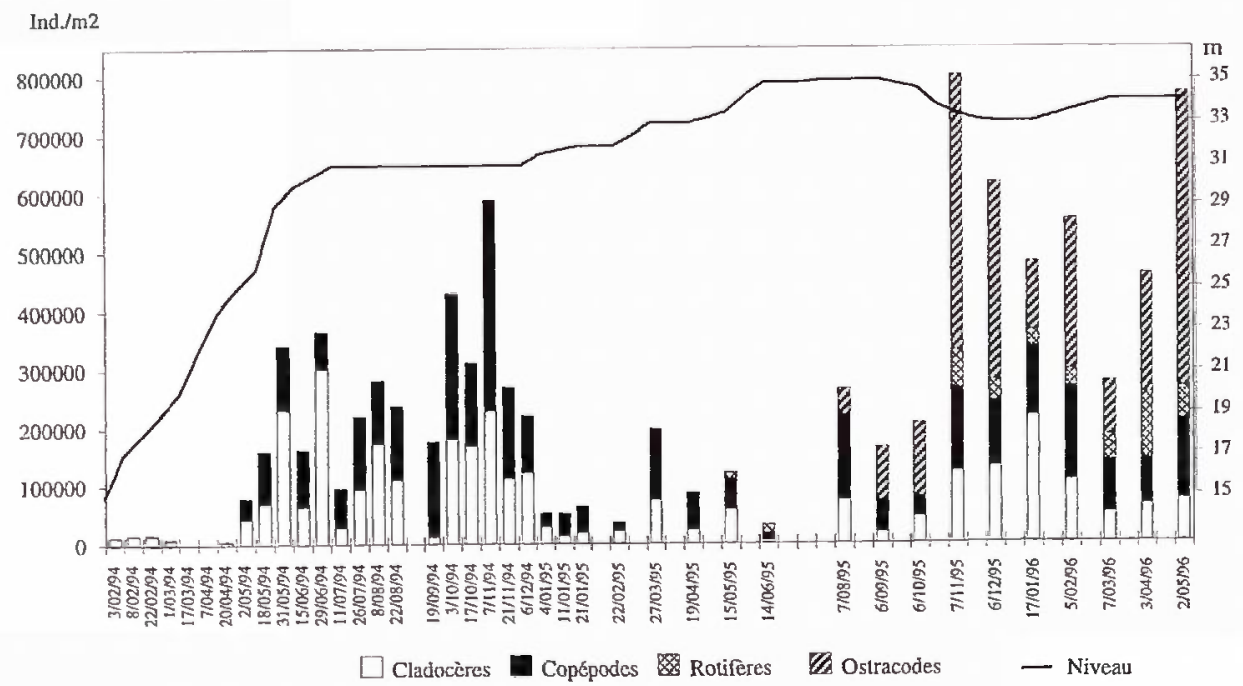

Fig. 4. - Evolution des principaux éléments du zooplancton depuis le début du remplissage (janvier 1994 - juin 1996), en relation avec le niveau de l'eau de la retenue, à Roche Génipa 1.

Fig. 4. - Roche Génipa 1. - development of zooplankton from the beginning of the filling phase (January 1994 - June 1996), related to water level. 
de la mise en eau : Bosminidae $\rightarrow$ cyclopoïdes $\rightarrow$ Daphniidae $\rightarrow$ calanoïd es $\rightarrow$ ostracodes.

\subsection{1.c Répartition horizontale de la faune pélagique}

\section{Répartition longitudinale}

Durant la phase de remplissage, il se forme des gradients horizontaux du fait des masses d'eau qui possèdent une dynamique propre, en matière de circulation, de flux et de temps de renouvellement de l'eau. II existe également un gradient dans la nature et dans la quantité des matériaux allochtones qui arrivent au lac. On observe alors une hétérogénéité qui influence la distribution des communautés planctoniques. II existe ainsi un gradient longitudinal de la queue jusqu'à la tête du réservoir. Heide (1982) à Brokopondo et Tundisi (1988) au Brésil définissent trois zones plus ou moins distinctes: le fleuve, la zone intermédiaire (de transition) et le lac. Ces zones sont notamment caractérisées par l'allure des courbes d'iso-concentrations en oxygène dissous (Richard, 1996). Heide (1982) observe différents peuplements planctoniques en fonction de ces zones. Afin de les distinguer durant la phase de remplissage, en fonction des entrées et des sorties d'eaux, plusieurs transects longitudinaux ont été effectués dans le réservoir de $\mathrm{Pe}$ tit-Saut à différentes dates. Celui de juin 1995 est présenté en exemple (fig. 5).

Ces transects longitudinaux nous permettent essentiellement de mettre en évidence l'avancée du front en période de fortes pluies, lorsque les débits sont importants comme en juin 1995. Lors de l'installation du zooplancton dans le lac, le milieu est tout d'abord colonisé par des petits cladocères (Bosminidae) puis apparaissent les petits cyclopoïdes. Lorsque nous analysons les différents transects longitudinaux, on assiste au même schéma que celui rencontré lors de la colonisation: dominance des cladocères au niveau de la queue de la retenue avec essentiellement des Bosminidae ou des Sididae puis les Daphniidae dominent à leur tour avec les calanoïdes. Ce schéma fonctionne sur toute la longueur des transects excepté pour la station de Petit-Saut dont l'hydrologie est plus souvent perturbée que dans les autres stations par la proximité des sorties d'eau (évacuateurs de fond, turbines,....).

Lorsque les apports sont moins importants, durant la saison sèche, le lac est dans une situation plus homogène et la zone de transition diminue.

Près du barrage, en aval de Génipa, des espèces détritivores comme les Bosminidae et les cyclopoïdes sont également bien représentées en réponse aux évacuations d'eau (de fond, de surface, de turbinage).

Fig. 5. - Transect longitudinal du 23-VI-95. A. - Zooplancton et chlorophylle a (données Vaquer) des 22 et 23-VI-95; B. - Détail des cladocères; C. - Détail des copépodes.

Fig. 5. - Horizontal plankton distribution - 23-VI-95. A. - Zooplankton and Chlorohyll a (Vaquer's data) 22 and 23-VI-95; B. - Cladocera; C. - Copepoda. 
A : Transect longitudinal du 23-VI-95

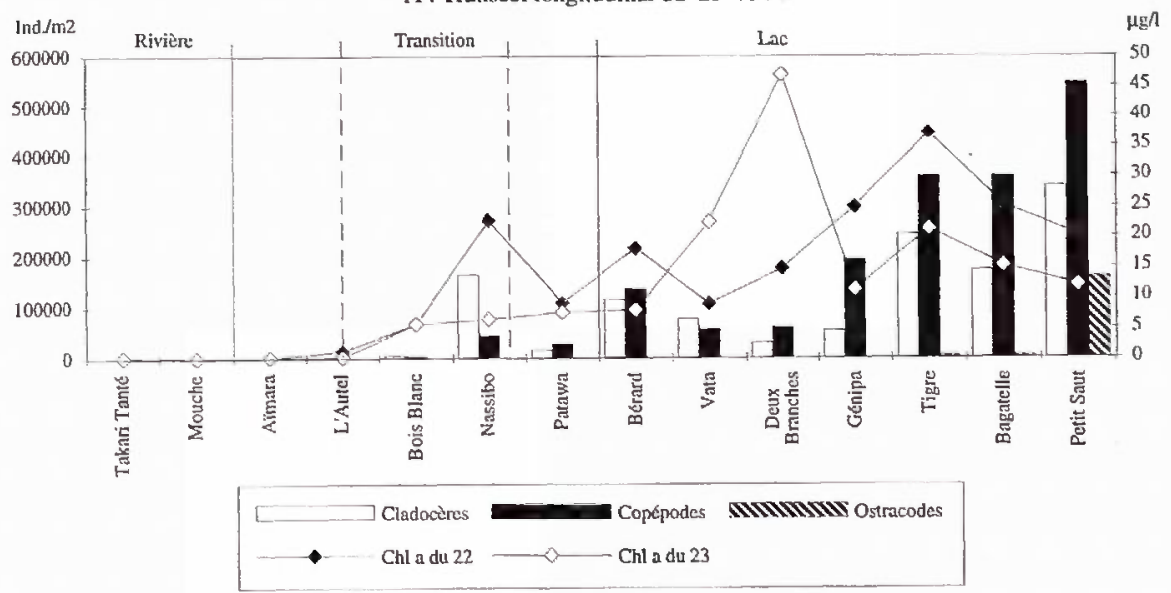

: Délimitations des trois zones selon les caractéristiques physico-chimiques.

. - . Délimitations des trois zones sclon les caractéristiques biologiques.
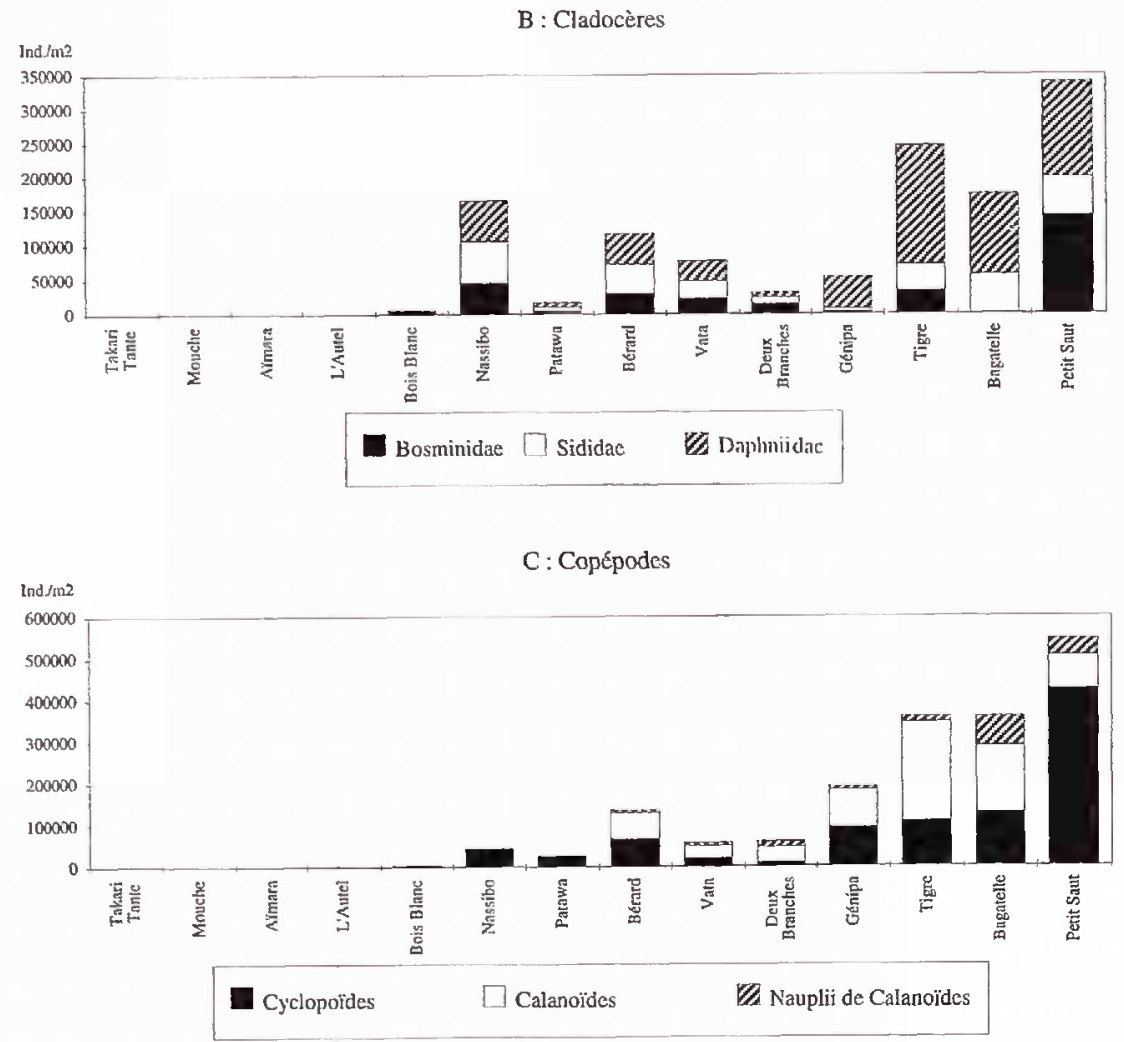


\section{Répartition transversale}

Pour des périodes similaires, nous avons comparé les peuplements pélagiques des stations axiales ou déboisées et ceux des stations non déboisées dans les différents sites prospectés. Ce type de comparaison est nécessaire dans la mesure où la zone de forêt est mise en eau plus tard que la zone axiale. De plus, la zone de forêt inondée représente un milieu plus fermé, plus encombré et donc moins éclairé. Ce biotope dont le fonctionnement peut se différencier de celui d'un lac occupe une superfi- cie beaucoup plus importante dans la retenue de Petit-Saut que la zone axiale correspondant aux anciens lits du fleuve et de ses affluents. Une estimation du poids des différents organismes collectés dans la retenue (Horeau, 1996) nous permet de faire des comparaisons de biomasse de la faune pélagique dans les différentes stations dans un intervalle de temps comparable; de juillet à décembre 1994 (tab. III).

Dans tous les cas, la biomasse des copépodes est supérieure à celle des cladocères (de 1,9 à 3,7 fois plus éle-

Tableau III. - Estimation de la biomasse du zooplancton dans les différentes stations prospectées dans la retenue de Petit-Saut. Les résultats, exprimés en $\mathrm{mg} \cdot \mathrm{m}^{-2}$, sont des moyennes des prélèvements effectués durant le second semestre de l'année 1994.

Table III. - Biomass of zooplankton in some stations of Petit-Saut dam. Results (mg. $\mathrm{m}^{-2}$ ) $=$ means (July - December 1994).

\begin{tabular}{|c|c|c|c|c|c|c|c|}
\hline & PS4 & PS2 & Génl & Gén2 & Gén4 & CBN1 & CBN2 \\
\hline Cladocères & 118,1 & 64,4 & 126,6 & 78,2 & 100,6 & 82,1 & 44,1 \\
\hline Bosminidae & 55,4 & 23,8 & 12,0 & 13,1 & 16,4 & 16,5 & 20,0 \\
\hline Sididae & 13,0 & 9,2 & 26,0 & 20,7 & 21,5 & 13,8 & 7,1 \\
\hline Daphniidac & 49,7 & 31,4 & 88,5 & 44,4 & 62,7 & 51,8 & 17,0 \\
\hline Copépodes & 118,5 & 123,7 & 273,0 & 168,1 & 244,5 & 220,8 & 162,5 \\
\hline Cyclopoides & 9,9 & 42,1 & 6,9 & 6,3 & 5,7 & 6,6 & 2,6 \\
\hline Nauplii & 0,0 & 0,3 & 0,0 & 0,0 & 0,0 & 0,0 & 0,0 \\
\hline $\begin{array}{l}\text { Adultes ct } \\
\text { copepodites }\end{array}$ & 9,9 & 41,8 & 6,9 & 6,3 & 5,7 & 6,6 & 2,6 \\
\hline Calanoïdes & 108,6 & 81,5 & 266,1 & 161,8 & 238,8 & 214,1 & 159,8 \\
\hline Nauplii & 5,8 & 2,5 & 5,8 & 7,7 & 10,7 & 7,8 & 4,3 \\
\hline $\begin{array}{l}\text { Adultes et } \\
\text { copepodites }\end{array}$ & 102,8 & 79,1 & 260,3 & 154,2 & 228,1 & 206,4 & 155,5 \\
\hline \multicolumn{8}{|l|}{ Diptères } \\
\hline Chaoboridae & 79,4 & 75,7 & 88,4 & 49,4 & 19,9 & 353,0 & 187,0 \\
\hline \multirow[t]{2}{*}{ Total } & 316,1 & 263,7 & 488,0 & 295,7 & 365,0 & 655,9 & 393,5 \\
\hline & $\begin{array}{l}\text { PS4 : Petit } \\
\text { déboiséce } \\
\text { PS2 : Petit }\end{array}$ & $\begin{array}{l}\text { zone axiale } \\
\text { zone boisée }\end{array}$ & $\begin{array}{l}\text { Gén1 : Roche G } \\
\text { Gén2 : Roche G } \\
\text { Gén4 : Roche G }\end{array}$ & $\begin{array}{l}\text { ipa, zone } \\
\text { ipa, zone } \\
\text { ipa, zone }\end{array}$ & $\begin{array}{l}\text { iale déboisée } \\
\text { iśce } \\
\text { boisée }\end{array}$ & $\begin{array}{l}\text { CBN1 : Crique } \\
\text { zone déboisée } \\
\text { CBN2: Crique } \\
\text { zone boisée }\end{array}$ & $\begin{array}{l}\text { ne Nouvelle, } \\
\text { ne Nouvelle, }\end{array}$ \\
\hline
\end{tabular}


vée) excepté pour la station déboisée de Petit-Saut, non représentative car perturbée par la proximité des sorties d'eau, où la biomasse des copépodes est proche de celle des cladocères.

Au total, la densité et la biomasse du zooplancton pélagique est meilleure en zone ouverte où le phytoplancton se trouve dans des conditions optimales pour une forte production. Ainsi, les phytoplanctonophages se situent préférentiellement dans ces zones ouvertes.

Dans le détail, les Bosminidae, les Macrothricidae et les copépodes sont en général mieux représentés dans les zones boisées car les débris de la forêt inondée dont ils se nourrissent sont abondants. Les Daphniidae et les calanoïdes plus phytoplanctonophages trouvent plus de nourriture dans les zones axiales et déboisées, où la photosynthèse est plus intense sans l'ombre des arbres.

II n'existe pas encore d'espèces héléoplanctoniques, différentes des espèces pélagiques, permettant d'identifier une zone littorale différente des zones axiales et boisées.

\subsubsection{La faune benthique}

La faune benthique se met en place beaucoup moins rapidement que la faune pélagique. Dans la retenue de Petit-Saut, le fond est anoxique et cette faune se situe essentiellement sur des supports (troncs d'arbre immergés) dans l'épaisseur d'eau oxygénée. Grâce aux substrats artificiels placés dans les différentes stations, plus de 29000 individus ont été récoltés durant la période de mai 95 à mai 96 . II s'agit de microcrustacés, de larves d'insectes, d'oligochètes et de nématodes (fig. 6).

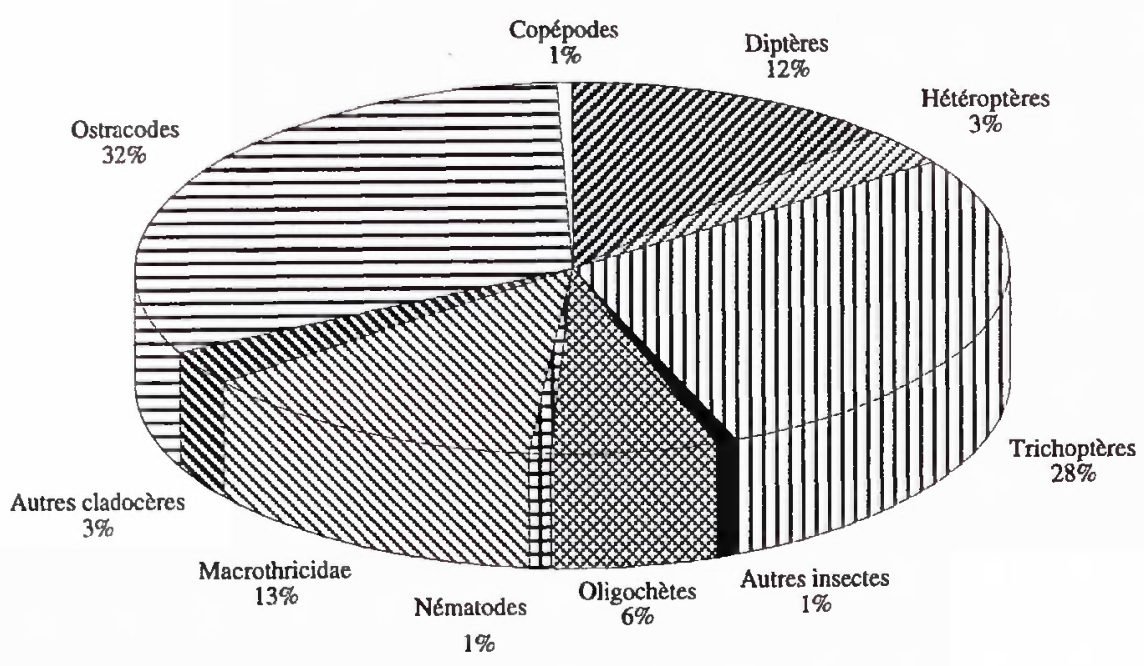

Fig. 6. - Pourcentages des différents invertébrés récoltés sur la totalité des substrats artificiels placés dans le lac.

Fig. 6. - Invertebrate rates collected on artificials substrate in Petit-Saut reservoir. 
Les microcrustacés sont essentiellement représentés par des ostracodes et des cladocères Macrothricidae. Ces Macrothricidae passent inaperçus aux vues des autres taxons dans les prélèvements de pleine eau où leur rencontre reste accidentelle.

Les larves d'insectes sont essentiellement constituées de trichoptères, de diptères dont $95,6 \%$ sont des Chironomidae et d'hétéroptères. D'autres taxons sont également présents comme les odonates, les éphémères ou les plécoptères. II faut noter, au dessus du plan d'eau, la présence d'un grand nombre d'imago de libellules qui font l'objet de collectes.

Cette faune représente actuellement une biomasse importante.

\subsubsection{La nourriture des poissons}

Les poissons représentent le dernier maillon de la chaîne alimentaire et peuvent avoir une valeur économique dans un écosystème aquatique tel que celui de Petit-Saut. C'est pour- quoi, l'équipe du laboratoire d'ichtyologie du centre INRA de Kourou (Boujard \& Rojas-Beltran, 1988a et b; Boujard et al., 1988 et 1990) puis du centre ORSTOM de Cayenne (Tito de Morais \& Planquette, 1991; Tito de Morais et al., 1995 ; Lauzanne et al., 1995; Mérona et al., 1996 a et b) étudient leur peuplement. Nous collaborons avec cette dernière équipe pour l'étude plus précise de la nourriture des poissons. Comme cela a été réalisé dans les criques (Horeau et al., 1996a) et dans les sauts (Horeau, 1996) avant la mise en eau, nous nous intéressons désormais à la nourriture des poissons du lac.

Depuis la fermeture du barrage, 2769 estomacs de 46 espèces de poissons (tab. IV) sont analysés afin d'évaluer les modifications dans le comportement alimentaire des poissons dues à la mise en eau.

Dans le fleuve, biotope non perturbé par la mise en eau, la majorité des aliments est constituée de végétaux et d'invertébrés terrestres (fig. 7). Ces

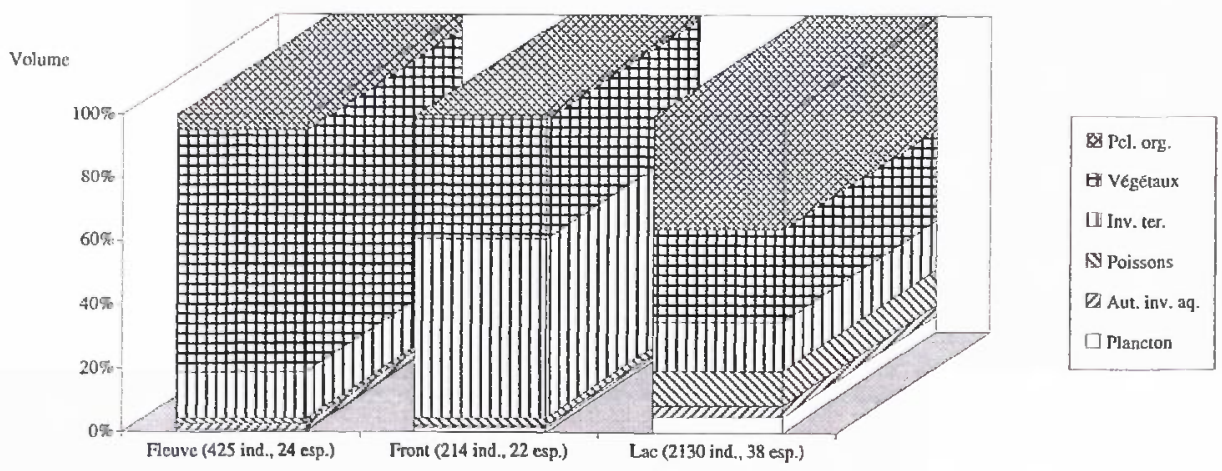

Fig. 7. - Pourcentages des aliments constituant la nourriture des poissons dans les différents biotopes.

Fig. 7. - Diet rates of fishes collected in different biotopes. 
Tableau IV. - Poissons dont les estomacs sont analysés et qui proviennent de l'amont de l'ouvrage depuis le début du remplissage.

Table IV. - Fishes collected upstream of dam from the beginning of the filling phase (stomac contents analysed).

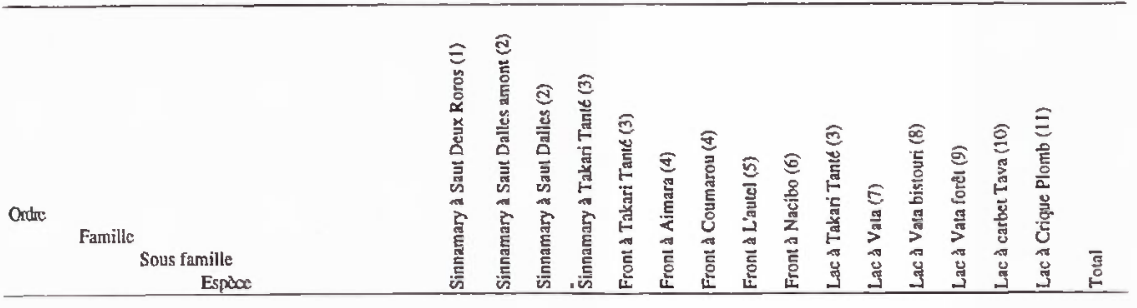

Cypriniformes

\begin{tabular}{|c|c|c|c|c|c|c|c|c|c|c|c|c|c|c|c|c|c|}
\hline \multirow{2}{*}{ Enģraulidac } & \multirow{3}{*}{ Anchovin surinamensis } & \multirow{3}{*}{-} & \multirow{3}{*}{ - } & \multirow[b]{2}{*}{ - } & \multirow[b]{2}{*}{ - } & \multirow{2}{*}{ - } & \multirow{2}{*}{-} & \multirow{2}{*}{ - } & \multirow[b]{2}{*}{ - } & \multirow{2}{*}{\multicolumn{2}{|c|}{-}} & \multirow[b]{2}{*}{-} & \multirow[b]{2}{*}{ - } & \multirow[b]{2}{*}{ - } & \multirow[b]{2}{*}{$=$} & \multirow[b]{2}{*}{1} & \multirow[b]{2}{*}{1} \\
\hline & & & & & & & & & & & & & & & & & \\
\hline Anostomidac & & & & & & & & & & & & & & & & & \\
\hline & Anostomus brevior & - & - & - & - & - & - & - & * & $=$ & 1 & - & $=$ & 1 & - & $=$ & 2 \\
\hline & Leporinus faciatus & - & - & - & 1 & - & - & - & - & - & - & - & $=$ & - & - & 1 & 2 \\
\hline & Leporinus friderici & 3 & 6 & 2 & 26 & 11 & - & - & - & - & 9 & - & 11 & 20 & 20 & 10 & 118 \\
\hline & Leporinus gossei & - & - & - & - & $!$ & - & - & - & - & 1 & - & 1 & 4 & - & $=$ & 7 \\
\hline & Leporinus granti & - & - & - & 2 & 2 & - & - & - & - & - & - & 2 & 3 & 1 & 1 & 11 \\
\hline & Leporinus pellegrini & - & - & - & $\mathbf{I}$ & - & - & - & - & $=$ & I & - & - & - & - & $\cdot$ & 2 \\
\hline
\end{tabular}

Characidae Bryconinae

Triportheus rotundatus

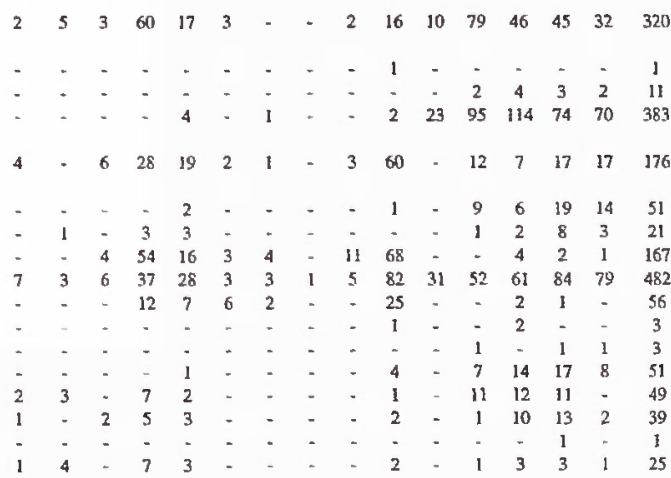

Curimatidac
Chilodontinac

Acestrorhynchus falcarus

Acrstrorhynchus microlepis

Charax gibbosus Stethaprioninac

Popsella orticularis

Teuragonopterinac

Astranax binaculatus

Astyanax polylegis

Bpiconops sp. 1

Biyconops sp.2

Bryconops.sp.3

Deuterodon sp.

Herrigrammus inilincatus

Moenlhawsia comme

Moenkhausia georgine

Moenuhausia oligolepis

Mocnlhausia sp.

Moenkhausia surinamensis

Chilodus annevei

Curimalinac

Curimara cyprinoides

Cyphocharax hellori

Cyphocharnx spilurus

Cyphocarar sp.

Hemiodidae

Hemiodontinac

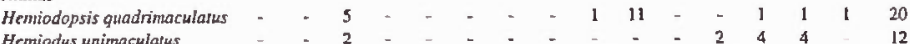

Sernssalmidae

Ayleus mombidalis

Rumphichtyidae fyleus iemetri

Eigennannia virescens

Siernopigus macrurus

Silunifomes

Auchenipteridac

\begin{tabular}{|c|c|}
\hline & $\begin{array}{l}\text { Aucheniplerus muchalis } \\
\text { Parauchenipterus galearus } \\
\text { Taria intermedia }\end{array}$ \\
\hline Doradidae & Doras carinatus \\
\hline Loticariidae & Hypostomus plecostomis \\
\hline Pimelodidac & $\begin{array}{l}\text { Pimelodella cristata } \\
\text { Pimelodella gracilis } \\
\text { Pimelodus ornatus }\end{array}$ \\
\hline
\end{tabular}

Perciformes

Cichlidas

Bivilmanchia bimaculnto

Cichlasoma bimaculatum

Geophagus surinamensis 
derniers évoluent dans la forêt environnante, soit au sol, soit dans les arbres. Ils se retrouvent "accidentellement " dans l'écosystème aquatique où ils sont appréciés par les poissons. Ce phénomène est d'autant plus marqué lorsque le couvert forestier est important comme c'est le cas au niveau des criques où la part des invertébrés terrestres atteint $65 \%$ des aliments des poissons (Horeau et al., 1996a). Les invertébrés aquatiques sont très peu représentés dans la nourriture des poissons du fleuve.

Au niveau du front, la part des invertébrés terrestres est très importante (plus de $56 \%$ des aliments). II faut rappeler que le front représente la limite entre le fleuve et le lac. Dans cette zone, la faune terrestre est touchée par la montée des eaux. Soit elle est noyée, directement disponible pour les poissons, soit elle se réfugie sur les arbres, ultérieurement disponible pour les poissons.

Dans le lac, le plancton (microcrustacés et larves de Chaoboridae), les macroinvertébrés de la forêt inondée (odonates, hétéroptères, trichoptères,...) qui représentent des ressources alimentaires potentielles importantes et les nombreux invertébrés terrestres des différentes strates de la forêt ennoyée (coléoptères, fourmis, termites, scorpions, annélides,....) sont consommés. Ces proies ne représentent pas un changement fondamental de nourriture pour les nombreux poissons identifiés dans le Sinnamary comme étant insectivores et omnivores, mais confirment l'opportunisme alimentaire de la plupart des poissons de cette région. Pour ce qui concerne le plancton, on constate que plusieurs espèces de poissons changent de régime alimentaire et deviennent, du moins en partie, zooplanctonophages dans la retenue. C'est le cas de Leporinus friderici (fig. 8) qui, dans le lac, consomme beaucoup d'invertébrés aquatiques dont des microcrustacés. De même, Triportheus rotundatus (Characidae) très fréquent dans le lac de barrage, se nourrit pour une grande part de zooplancton dans la retenue (fig. 9).

Dans le temps, on observe des variations dans la proportion des différents aliments en fonction des disponibilités du milieu. Nous prenons le cas de Triportheus rotundatus dans les stations du lac (fig. $10 \mathrm{~A}$ ), en relation avec le niveau de la retenue (fig. $10 \mathrm{~B}$ ).

Au début de la mise en eau, les invertébrés terrestres représentent $80 \%$ des aliments chez ce poisson. Lors de l'inondation de la forêt primaire, les nombreux invertébrés terrestres surpris par les eaux sont consommés par les poissons.

Très rapidement, les invertébrés pélagiques se développent dans la retenue. Au début de la mise en eau, il s'agit essentiellement de petits organismes (les microcrustacés). Malgré sa petite taille, le zooplancton participe à la nourriture de ce poisson (jusqu'à $100 \%$ en mai 94 ).

Ensuite, la part relative des invertébrés aquatiques et terrestres oscille. Ces variations sont à mettre en relation avec le niveau de la retenue. 


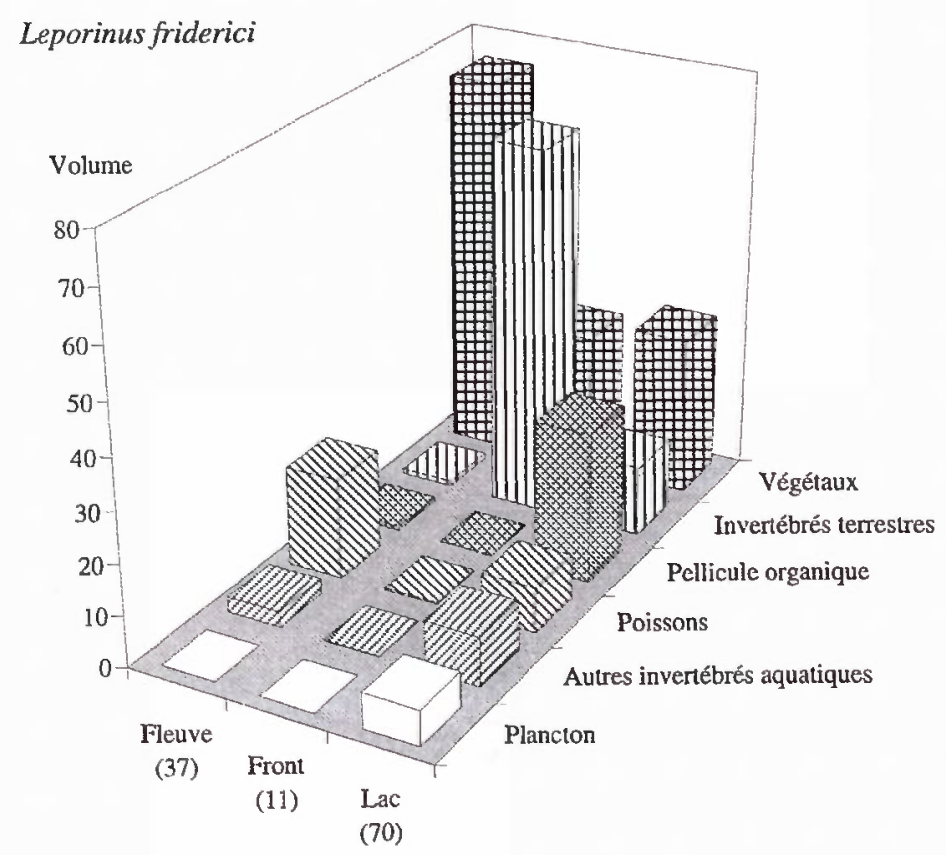

Fig. 8. - Pourcentages des aliments constituant la nourriture de Leporinus friderici dans différents biotopes. (). - nombre de poissons analysés.

Fig. 8. - Diet rates of Leporinus friderici in different biotopes. (). - fishes number analysed.

Dans le lac, lorsque les eaux montent, des invertébrés terrestres sont à nouveaux disponibles, par ennoiement des zones de marnage et des nombreux troncs sur lesquels des individus se réfugient. Les fortes pluies provoquent également la chute de nombreuses branches riches en invertébrés terrestres. Autour du lac, de nombreux individus de la forêt environnante tombent également dans le lac, entraînés par les fortes pluies. C'est pourquoi, lors de ces montées du niveau de la retenue liées à l'exploitation du barrage, comme de janvier à juin 1995 ou de janvier à avril 96, la part des invertébrés terrestres est très importante. En contre partie, lorsque le niveau se stabilise ou baisse, la part des invertébrés terrestres diminue et est remplacée par des invertébrés aquatiques essentiellement constitués de zooplancton (microcrustacés et Chaoboridae).

Ainsi, au niveau du lac de barrage, il est important de souligner :

- la disparition rapide de la faune d'eau courante qui était principalement constituée de larves d'éphémères, de trichoptères, de diptères, d'odonates,... ainsi que de crustacés décapodes,

- la rapidité de la production biologique lacustre puisque dès les premiers mois de la mise en eau, le 


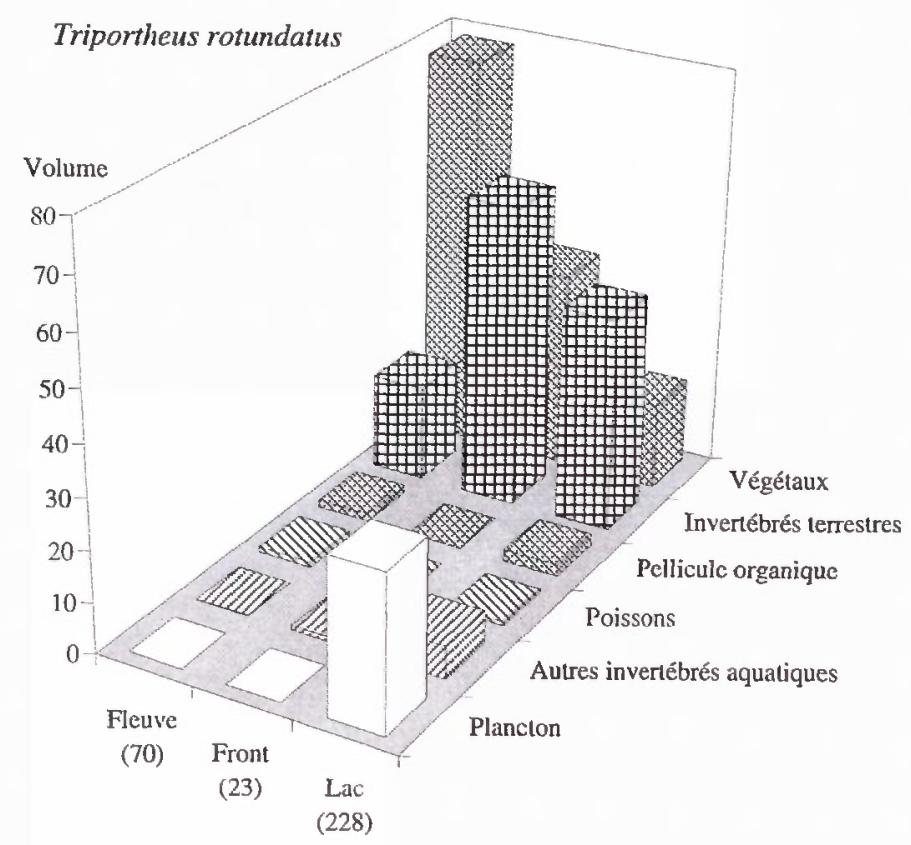

Fig. 9. - Pourcentages des aliments constituant la nourriture de Triportheus rotundatus dans diffêrents biotopes. (). - nombre de poissons analysés.

Fig. 9. - Diet rates of Triportheus rotundatus in different biotopes. (). - fishes number analysed.

zooplancton est très dense dans la retenue (plus de 80000 ind. $\mathrm{m}^{-2}$ en amont immédiat de l'ouvrage, dès le premier mois de remplissage),

- la diversité du zooplancton dès le début de la mise en eau,

- la forte densité du zooplancton, tout d'abord des cladocères détritivores, en relation avec les quantités de débris organiques puis des espèces planctonophages avec le développement du phytoplancton,

- la bonne utilisation de ces nouvelles ressources alimentaires lacustres par les poissons. Alors qu'il est quasiment absent de l'alimentation des poissons du fleuve Sinnamary et de ses criques, le zooplancton est désormais consommé dans le lac $(15 \%$ dont $3 \%$ de microcrustacés et $12 \%$ de larves de Chaoboridae), de même que les invertébrés benthiques comme les larves de trichoptères $(4 \%)$, les hétéroptères $(4 \%)$, les odonates $(2 \%)$ et autres $(3 \%)$.

\subsection{L'aval du barrage}

Le tronçon aval reste un écosystème d'eau courante mais son alimentation n'est plus constituée exclusivement d'eaux fluviales mais principalement d'eaux lacustres. Les invertébrés d'eau courante provenant 

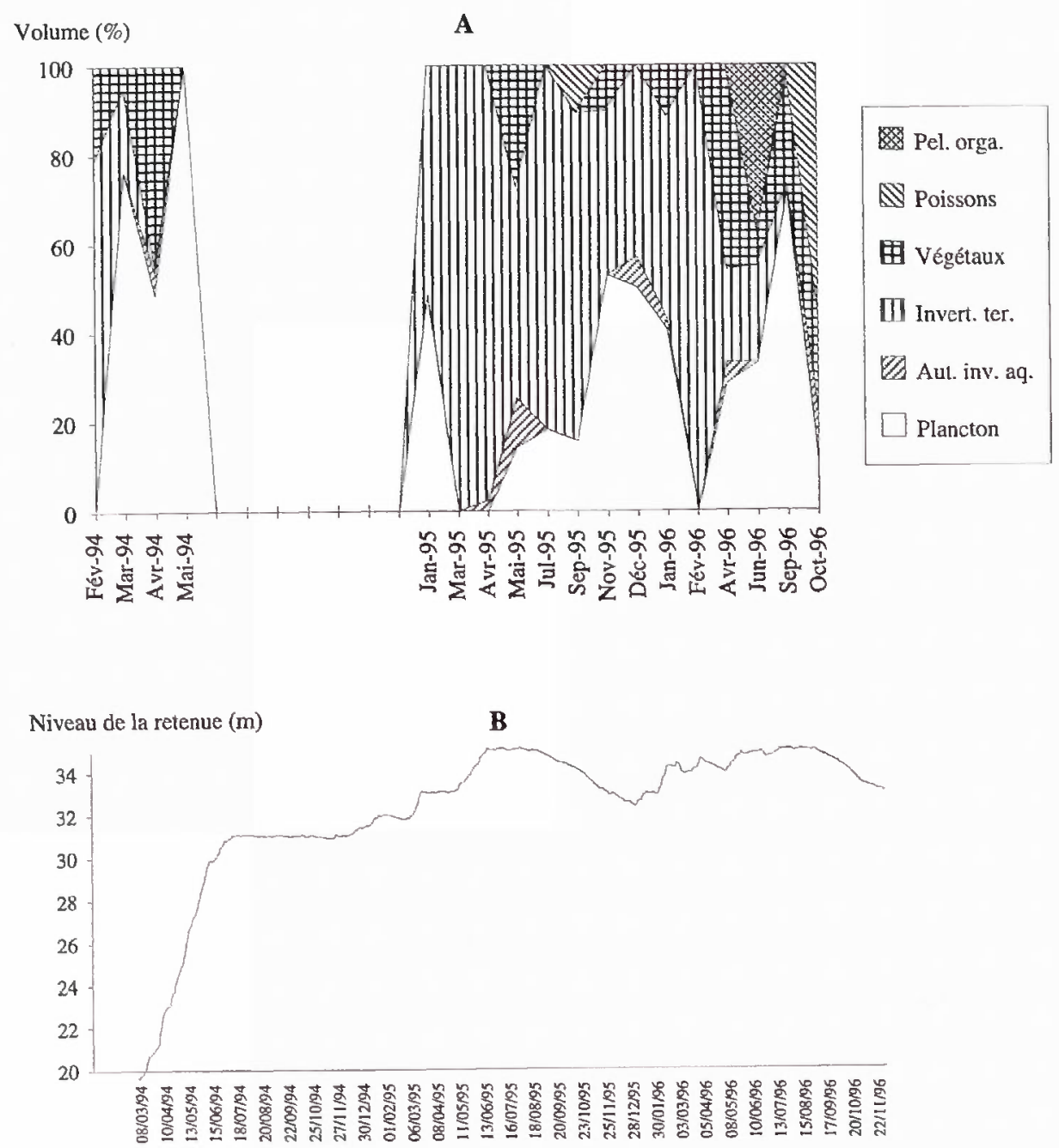

Fig. 10. - Evolution des aliments constituant la nourriture de Triportheus rotundatus depuis le début de la mise en eau, dans les stations du lac (A), en relation avec le niveau de la retenue (B).

Fig. 10. - Development of diet rates of Triportheus rotundatus from the beginning of the filling phase, in reservoir stations (A) in relation with the level of the dam (B).

essentiellement de la dérive de la faune concentrée dans les sauts (Horeau, 1996) disparaissent puisque les sauts ont été recouverts par les eaux du lac. La seule barre rocheuse en aval de l'ouvrage (Kerenroch) pouvant abritée cette faune de rapide est fortement perturbée par la qualité des eaux restituées par le barrage. Actuellement, les invertébrés sont remplacés par le zooplancton pélagique de la retenue qui se retrouve en dérive 
dans le fleuve. Cette faune d'eau courante principalement constituée des microinvertébrés pélagiques du lac ainsi que la faune benthique vivant dans le sable sont directement influencées par la qualité des eaux de la retenue et par la gestion de l'ouvrage.

\subsubsection{La faune d'eau courante}

D'un point de vue quantitatif, la microfaune est en moyenne quarante fois plus dense, en nombre d'individus par unité d'échantillonnage, depuis le début de la mise en eau (Horeau, 1996).

D'un point de vue qualitatif (fig. 11), les microcrustacés dominent largement dans la masse d'eau. On retrouve les cladocères Bosminidae, Daphniidae, Sididae du lac. Les Ma- crothricidae, peu représentés dans la faune pélagique du lac, se maintiennent relativement bien dans le fleuve, maintien peut-être dû aux fortes teneurs en matières organiques que ces organismes apprécient. Les copépodes cyclopoïdes, calanoïdes ainsi que les Chaoboridae se retrouvent également en dérive dans le fleuve. Dans le temps, les variations des invertébrés de l'aval dépendent directement des variations observées dans les peuplements du lac.

Comme cela a été mis en évidence avec certains paramètres physico-chimiques (Richard, 1996) et avec les teneurs en méthane (Galy-Lacaux, 1996), la faune de l'aval montre qu'une partie des eaux turbinées provient de l'épilimnion. Effectivement, lorsque l'ouvrage ne restitue que des

A
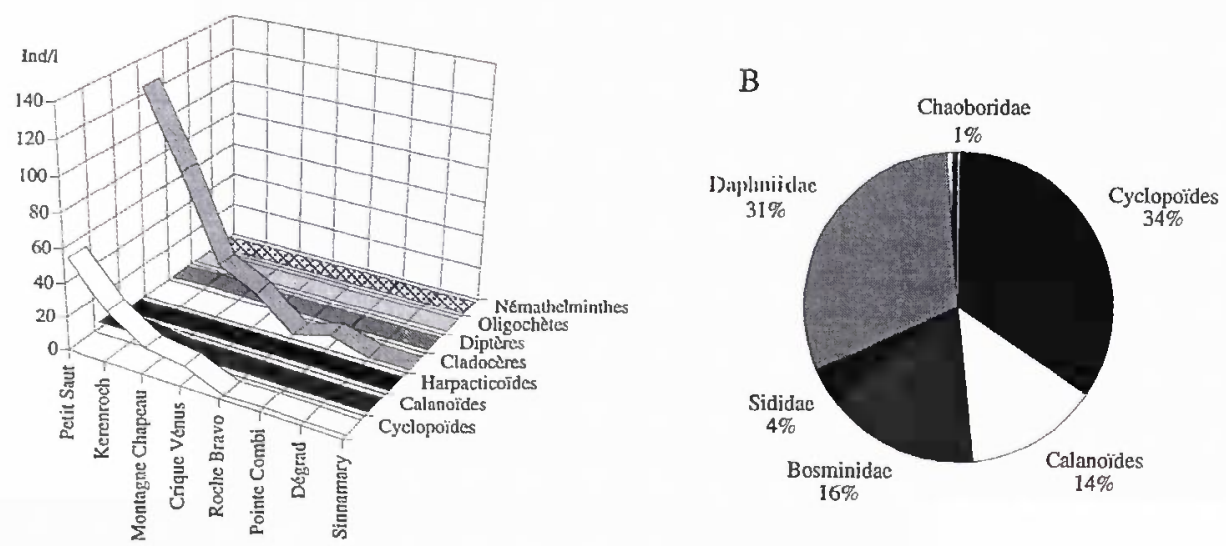

Fig. 11. - Répartition des peuplements d'invertèbrés aquatiques à l'aval de l'ouvrage de Petit-Saut, le 02 juin 1994 (A), directement liée aux peuplements d'invertébrés aquatiques de la station lacustre Petit Saut 4 , à la même époque (B).

Fig. 11. - Distribution of aquatic invertebrate populations downstream of Petit-Saut dam, on the $2^{\text {nd }}$ June 1994 (A), related to the fauna in the lake at Petit Saut 4 station, during the same period (B). 
eaux turbinées (prise d'eau au fond du lac), les eaux à l'aval contiennent de la faune épilimnique.

La faune en dérive dans l'axe du fleuve reste vivante jusqu'à l'estuaire.

\subsubsection{La faune benthique du sable}

Dans le substrat sableux (fig. 12), avant la mise en eau, les cyclopoïdes dominaient à Kerenroch, station située à $8 \mathrm{~km}$ en aval de l'ouvrage. Ces invertébrés évoluaient dans un milieu pauvre en nutriments. Depuis la mise en eau de la retenue, les harpacticoïdes dominent les peuplements du sable dans ce secteur aval. Ils s'agit d'harpacticoïdes Parastenocaridae de forme très effilée, généralement cantonnés aux milieux psammiques (Dussart, 1967).
Depuis le début de la mise en eau, la faune du fond évolue. A Kerenroch, la faune d'invertébrés benthiques (fig. 13), constituée essentiellement d'harpacticoïdes, de némathelminthes et d'oligochètes, est relativement dense à partir de juin 1994. Durant l'été 1994, les apports en matières organiques provenant du barrage sont propices à son développement. Mais à partir de septembre 1994, les particules organiques colmatent le substrat qui devient anoxique et la faune benthique se fait rare. Mai 1995 marque le retour du développement de la faune d'invertébrés benthiques. Ces variations peuvent être en partie explicables par les teneurs en carbone organique mesurées dans le sable qui tendent à diminuer à partir d'avril 1995.

B $(n \approx 32000)$

$A(n=100)$

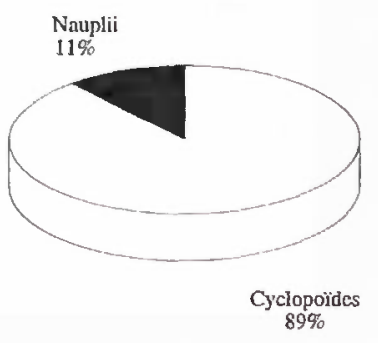

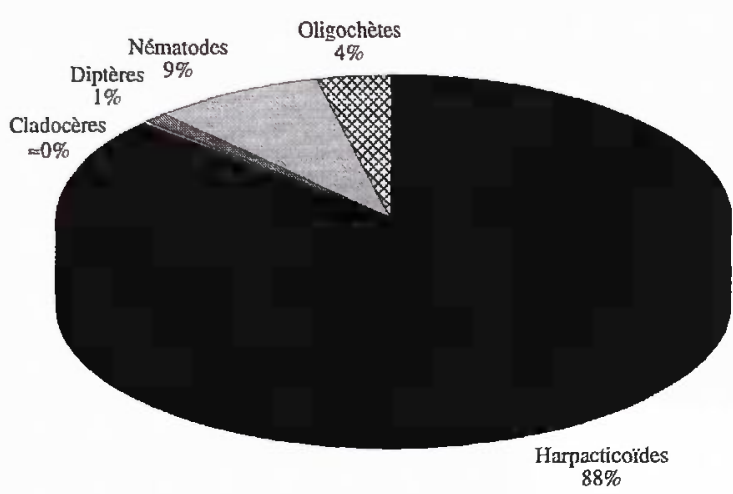

Fig. 12. - Peuplement d'invertébrés aquatiques du sable, en aval de l'ouvrage, après la fermeture du barrage (janvier 1994 - juin 1995).

Fig. 12. - Aquatic invertebrate populations downstream of Petit Saut dam in sand substrate before and after the completion of the dam (January 1994 - June 1995). 


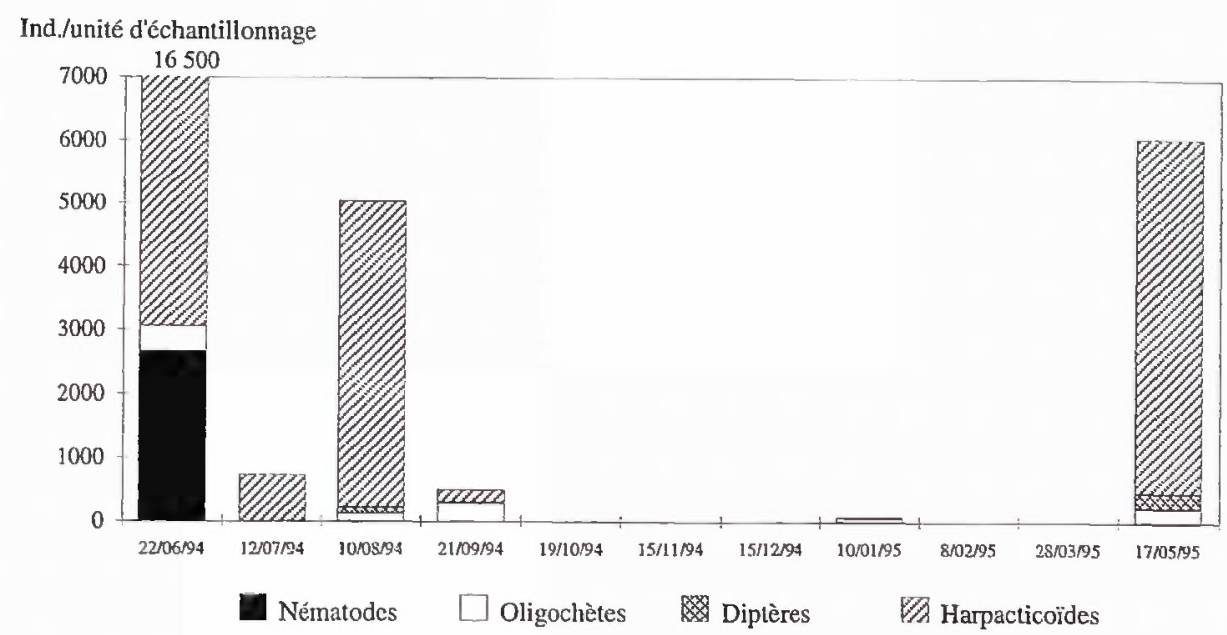

Fig. 13. - Evolution des populations d'invertébrés aquatiques du sable, à l'aval du barrage de Petit-Saut, durant la mise en eau (janvier 1994 - juin 1995).

Fig. 13. - Development of aquatic invertebrate populations downstream of the Petit-Saut dam, in the sand substrate, during the filling phase (January 1994 - June 1995).

Contrairement aux observations effectuées à l'aval d'ouvrages hydroélectriques amazoniens, comme à Curuá-Una, aucune accumulation de décapodes bloqués dans leur migration n'a été observée à l'aval du barrage de Petit-Saut.

Ainsi, les points essentiels à retenir pour l'aval de la retenue sont:

- le remplacement de la faune d'eau courante par la faune lacustre de la retenue en dérive dans le tronçon aval,

- l'enrichissement de la faune benthique par la matière organique provenant du lac,

- l'influence des qualités physicochimique et biologique des eaux du lac ainsi que de la gestion de l'ouvrage sur les invertébrés aquatiques de l'aval,

- la confirmation qu'il n'existe pas de migration d'invertébrés aquatiques susceptible d'être perturbée par le barrage.

\section{CONCLUSIONS ET PERSPECTIVES}

Nos travaux sur les invertébrés aquatiques nous ont permis d'apporter des éléments nouveaux sur le fonctionnement biologique de l'écosystème du fleuve Sinnamary avant l'implantation du barrage ainsi que sur le fonctionnement biologique de la re- 
tenue et de son tronçon aval. Nous mettons notamment en évidence que des invertébrés aquatiques comme les microcrustacés qui constituent pour l'instant l'essentiel de la faune lacustre, répondent très rapidement aux variations des paramètres physicochimiques des eaux dans lesquelles ils évoluent. Le suivi de ces organismes est important à court et moyen terme pour répondre à plusieurs questions sur le fonctionnement de l'épilimnion où est concentrée l'activité biologique.

Nous poursuivons nos efforts pour évaluer la diversité des invertébrés aquatiques en collaboration avec des spécialistes de différents groupes comme Defaye pour les copépodes, Machet pour les odonates, Martens pour les ostracodes, Peters pour les éphémères ou Pourriot pour les rotifères. D'autres groupes échantillonnés et conservés comme celui des cladocères pourront faire l'objet d'une étude systématique plus complète. Ces déterminations nous permettrons d'effectuer des comparaisons plus précises sur la diversité d'avant et d'après mise en eau.

Dans la zone pélagique, il sera intéressant d'étudier la micro-répartition du zooplancton pour vérifier si l'épilimnion fonctionne de façon autonome par rapport à l'hypolimnion, ce qui est le cas dans les autres lacs oligomictiques naturels de la zone intertropicale. Il faudra également évaluer, en collaboration avec les études sur le phytoplancton (Vaquer, 1997), la part des algues consommées par les invertébrés et celle qui sédimente et augmente le stock de matière organique hypolimnique.

Le bon fonctionnement biologique de l'épilimnion pourrait expliquer en partie que certains effets négatifs annoncés n'ont pas ou peu été observés; ainsi, par exemple, la qualité des eaux des criques confinées est restée semblable à celle des eaux de la zone axiale renouvelées plus rapidement. De même, l'effet néfaste du découpage des rives ne s'est pas encore manifesté par le développement de macrophytes (Blake, 1997), lesquelles ne trouvent peut-être pas suffisamment d'éléments nutritifs, consommés plus rapidement par le phytoplancton.

Un travail complémentaire pourra également être réalisé sur divers points d'écophysiologie comme la résistance respective des différents groupes de zooplancton aux déficits en oxygène dissous par le biais d'une expérimentation en laboratoire, pour expliquer la micro-répartition verticale du zooplancton ainsi que la recolonisation très rapide du zooplancton après les phases de mélange partiel lors des fortes pluies.

Dans la zone de forêt inondée, une étude de la biomasse des macroinvertébrés en diversifiant les méthodes de prélèvements permettrait de définir le rôle respectif des macroinvertébrés et du zooplancton dans la consommation de la matière organique d'une part et dans l'alimentation des poissons d'autre part. Cette étude permettrait également de préciser le rôle des invertébrés aquatiques dans cette zone, afin de définir si la forêt inondée 
fonctionne comme la zone littorale des lacs sous d'autres latitudes puisque à Petit-Saut, la zone littorale est biologiquement absente pour l'instant.

Dans la zone de marnage qui représente un tiers de la superficie du plan d'eau durant la phase exondée, une forte production primaire terrestre pourrait apporter un surcroît de matière organique mais dans le cas le plus probable d'une faible repousse, la minéralisation de la matière organique morte pourrait en diminuer le stock dans la retenue.

Des estimations complémentaires de biomasses, notamment de la faune benthique dans la retenue, permettront de comparer les biomasses d'invertébrés aquatiques disponibles avant la mise en eau dans le fleuve, les sauts, les criques et les pripris, avec celles disponibles actuellement sur l'ensemble de la retenue.

A l'aval, en plus du suivi de la microfaune dans la masse d'eau et dans le sable, il faudrait vérifier le maintien des populations de décapodes par des méthodes de prélèvement mieux adaptées ainsi que l'absence ou non de mouvements migratoires, et enfin poursuivre notre effort sur l'alimentation des poissons moins connue dans cette portion du fleuve.

\section{REMERCIEMENTS}

Cette étude a été financée par EDF dans le cadre d'un contrat HYDRE$\mathrm{CO} / \mathrm{EDF}-\mathrm{CNEH}$. Nous tenons à remercier toutes les personnes qui y ont contribué, notamment les spécialistes qui ont déterminé le matériel, D. Defaye, $P$. Machet, K. Martens, W.L. Peters, R. Pourriot et $\mathrm{J}$. Rey, ainsi que l'équipe technique du Laboratoire Environnement de Petit-Saut, R. Aboikoni, L. Guillemet et P. Raverat.

\section{RÉFÉRENCES BIBLIOGRAPHIQUES}

Blake G., 1997. Rapport de mission sur le développement de la végétation aquatique et semi-aquatique sur la retenue de Petit-Saut. Hydroécol. Appl. 9 (1/2) pp. 195-209.

Boujard T. \& Rojas-Beltran R., 1988a. Zonation longitudinale du peuplement ichtyque du fleuve Sinnamary (Guyane française). Rev. Hydrobiol. Trop., 21 : 47-61.

Boujard T. \& Rojas-Beltran R., 1988b. Description des captures au filet tramail sur le cours supérieur du Sinnamary (Guyane française). Rev. Hydrobiol. Trop., 21 : 349-356.

Boujard T., Le Bail P.Y. \& Planquette P., 1988. Données biologiques sur quelques espèces continentales de Guyane Française d'intérêt piscicole. Aquat. Living Resour., 1: 107-113.

Boujard T., Sabatier D., Rojas-Beltran R., Prevost M.F. \& Renno J.F., 1990. The food habits of three allochtonus feeding Characoids in French Guiana. Rev. Ecol. (Terre Vie), 45: 247-257.

Bournaud M., Tachet H. \& Roux A.L., 1987. The effects of seasonal and hydrological influences on the macroinvertebrates of the Rhône river. II - Ecological aspects. Archiv. Hydrobiol., Suppl. 76 (1/2) : 25-51.

Cereghino R. \& Lavaraller P., 1996. Influence of hydropeaking on the structure and dynamics of invertebrates populations in a mountain stream. $2^{\text {nd }}$ 
Intern. Symp. Hab. Hydrolics, Juin, Québec, (vol. A) : 699-710.

Décamps H., Capblancq J., Casanova $\mathrm{H}$. \& Tourenq J.N., 1987. Hydrobiology of some regulated rivers in the SouthWest of France. Ecol. Regul. Sream, (volume) : 273-287.

Dumont H., Carrel (G.), Le Coarer Y., Rivier B. \& Suard G., 1996. Towards the notion of trophic value. Concepts and approches. The brown trout example (Salmo truta). $2^{\text {nd }}$ Intern. Symp. Hab. Hydrolics, Juin, Québec, (Vol. B) : 283297.

Dussart B., 1967. Tome 1 : Calanoïdes et Harpacticoïdes. Les copépodes des eaux continentales d'Europe Occidentale, N. Boubée \& Cie ed., Paris. $500 \mathrm{p}$.

Falótico M.H.B., 1993. Característica limnológicas e aspectos da composição e distribuiçāo da comunidade zooplanctônica em sua fase de enchimento (reservatório de Samuel Rondônia). MSc. Thesis USP, São Paulo. $195 \mathrm{p}$.

Galy-Lacaux C., 1996. Modifications des échanges de constituants mineurs atmosphériques liées à la création d'une retenue hydroélectrique. Impact des barrages sur le bilan du méthane dans l'atmosphère. Thèse Univ. Paul Sabatier de Toulouse. $210 \mathrm{p}$.

Grégoire A., 1982. Contribution à l'étude hydrobiologique d'une rivière aménagée: le Verdon. Les lacs de barrage et les tronçons de cours d'eau à débit régulé. Cahier du laboratoire d'hydrobiologie de Montereau. 13: 1-172.

Heide J. Van Der, 1982. Filling phase limnology of man-made lake in the humid tropics. Lake Brokopondo, Offsetdrukkerij Kanter B.V. ed., Alblasserdam. $428 \mathrm{p}$.

Horeau V., 1996. La mise eau de la retenue hydroélectrique de Petit-Saut (Guyane). Hydrobiologie 1 - du fleuve Sinnamary avant la mise en eau, 2 de la retenue pendant la mise en eau,
3- du fleuve en aval. Thèse Univ. AixMarseille I. $250 \mathrm{p}$.

Horeau V., Cerdan P., Champeau A. \& Richard S., 1996a. Importance des apports exogènes dans le régime alimentaire de quelques poissons de " criques » du bassin versant du fleuve Sinnamary (Guyane française). Rev. Ecol. (Terre Vie), 51 : 29-41.

Horeau V., Champeau A., Grégoire A. \& Cerdan P., 1996b. Hydrological aspect of impouding at Petit-Saut (French Guiana). $2^{\text {nd }}$ Intern. Symp. Hab. Hydrolics, Juin, Québec, (Vol A) : 3748.

Horeau V. \& Richard S., 1992. Etat d'avancement des travaux sur l'hydrobiologie du bassin versant du fleuve Sinnamary (chimie, biologie). Rapport Univ. de Provence-HYDRECO-EDF, Petit Saut. 175 p.

Lauzanne L., Mérona B. de, Ponton D., Bron J.C., Raffray J., Mallet A. \& Brehm N., 1995. Présentation des résultats concernant les études ichtyologiques menées par l'ORSTOM. C.R. Com. Sc., J. Env., $5:$ 91-109.

Mérona B. de, Ponton D., Géguan J.F., Merigoux S., 1996a. Structure et biologie des peuplements ichtyques du fleuve Sinnamary en Guyane. Rap. Interm. ORSTOM - EDF, $45 \mathrm{p}$.

Mérona B. de, Ponton D., Géguan J.F., Merigoux S., 1996b. Situation des communautés de poissons dans le Sinnamary barré, un an et demi après la fermeture du barrage. C.R. Com. Sc., J. Env., 6 : 90-97.

Parasiewicz P., Schmutz S. \& Moog O., 1996. The effects of managed hydropower peaking on the physical habitat, benthos and fish fauna in the Bregenzcrach, a nival 6 th order river in Austria. $2^{\text {nd }}$ Intern. Symp. Hab. Hydrolics, Juin, Québec, (Vol. A): 685-697.

Pourriot R., 1996. Rotifers from Petit-Saut reservoir (French Guyana), with the description of a new taxon. Hydrobiologia, 331 : 43-52. 
Pourriot R., Horeau V., Rougier C., 1997. Filinia (Rotifera, Monogononta) populations in french Guyana: $F$. novaezealandiae, $F$. saltator with remarks on the variability of some characters. Arch. Hydrobiol. 139 (4) : 563-575.

Richard S., 1996. La mise eau de la retenue hydroélectrique de Petit-Saut (Guyane). Physico-chimie $1-d u$ fleuve Sinnamary avant la mise en eau, 2 - de la retenue pendant la mise en eau, $3-$ du fleuve en aval. Thèse Univ. Aix-Marseille I. 278 p.

Richard S., 1997. Evolution de la qualité physico-chimique de l'eau de la retenue depuis le début de la mise en eau du barrage de Petit-Saut. Hydroécol. Appl. $9(1 / 2):$ pp..

Segers H. \& Pourriot R., 1997. On a new and puzzling american rotifer (Rotifera : Monogononta, Lecanidae). Journal of Natural History, 31 : 383-388.
Tito de Morais L. \& Planquette P., 1991. Peuplements ichtyologiques du haut Sinnamary. Guyane Française. Rapport EDF, GP 1508-90073, 49 p.

Tito de Morais L., Lointier M. \& Hoff M., 1995. Extent and role for fish populations of riverine ecotones along the Sinnamary river (French Guiana), Hydrobiologia, 303: 163-179.

Tundisi J.G., 1988. Limnologia e manejo de represas. Sao Carlos. EESCSP/CHREA/ACIESP, $506 \mathrm{p}$.

Vaquer A., 1997. Distribution spatio-temporelle du phytoplancton dans le réservoir de Petit-Saut (Guyane). Hydroécol. Appl. 9 (1/2) : pp. 169-193.

Ward J.V. \& Stanford J.A., 1987. The ecology of regulated streams past accomplishments and directions for future research. Regulated streams: advances in ecology. Graig J.F. \& Kemper J.B. ed., New York: 391-409. 